\title{
DFT Study on the Deglycosylation of Methylated, Oxidized, and Canonical Pyrimidine Nucleosides in Water: Implications for Epigenetic Regulation and DNA Repair
}

Ye Eun (Rebecca) Jeong, Stefan A. P. Lenz, Stacey D. Wetmore*

Department of Chemistry and Biochemistry, University of Lethbridge, 4401 University Drive

West, Lethbridge, Alberta, Canada T1K $3 M 4$

\section{SUPPORTING INFORMATION}

(28 Pages)

\section{Table of Contents}

Table S1. Calculated Gibbs Energy Barriers $\left(\Delta^{\ddagger} G\right)$ and Reaction Energies $\left(\Delta G_{\mathrm{rxn}}, \mathrm{kJ} / \mathrm{mol}\right)$ for the Hydrolytic Deglycosylation of the $5 \mathrm{ca}-\mathrm{dC}$ Nucleoside Facilitated by the $\mathrm{HO}^{-}$Nucleophile ${ }^{a}$ with Different Conformations of the C5 Substituent.

Figure S1. Stationary points (reactant complexes (RC), transition states (TS) and product complexes (PC)) along the deglycosylation of the $5 \mathrm{ca}-\mathrm{dC}$ nucleoside with different $\mathrm{C} 5$ substituent conformations facilitated by the $\mathrm{HO}^{-}$nucleophile. S4

Coordinates for the TS along the deglycosylation of $\mathrm{dC}$ facilitated by the $\mathrm{HO}^{-}$nucleophile....... S4 Coordinates for the TS along the deglycosylation of $5 \mathrm{~m}-\mathrm{dC}$ facilitated by the $\mathrm{HO}^{-}$nucleophile. S5 Coordinates for the TS along the deglycosylation of $5 \mathrm{hm}-\mathrm{dC}$ facilitated by the $\mathrm{HO}^{-}$nucleophile.

Coordinates for the TS along the deglycosylation of $5 \mathrm{f}$ - $\mathrm{dC}$ facilitated by the $\mathrm{HO}^{-}$nucleophile...S6 Coordinates for the TS along the deglycosylation of $5 \mathrm{ca}-\mathrm{dC}(\mathrm{C} 4)$ facilitated by the $\mathrm{HO}^{-}$ nucleophile.

Coordinates for the TS along the deglycosylation of $5 \mathrm{ca}-\mathrm{dC}\left(\mathrm{O5}^{\prime}\right)$ facilitated by the $\mathrm{HO}^{-}$ nucleophile.

Coordinates for the TS along the deglycosylation of $5 \mathrm{ca}-\mathrm{dC}(\mathrm{N} 4)$ facilitated by the $\mathrm{HO}^{-}$ nucleophile.

Coordinates for the TS along the deglycosylation of $5 \mathrm{ca}-\mathrm{dC}(\mathrm{C} 6)$ facilitated by the $\mathrm{HO}^{-}$ nucleophile.

Coordinates for the TS along the deglycosylation of dU facilitated by the $\mathrm{HO}^{-}$nucleophile..... S10 Coordinates for the TS along the deglycosylation of dT facilitated by the $\mathrm{HO}^{-}$nucleophile......S11 Coordinates for the TS along the deglycosylation of $5 \mathrm{hm}$-dU facilitated by the $\mathrm{HO}^{-}$nucleophile. 
Coordinates for the TS along the deglycosylation of $\mathrm{dC}$ facilitated by the $\mathrm{HO}^{-} \ldots \mathrm{H}_{2} \mathrm{O}$ nucleophile.

Coordinates for the TS along the deglycosylation of $5 \mathrm{~m}-\mathrm{dC}$ facilitated by the $\mathrm{HO}^{-} \cdots \mathrm{H}_{2} \mathrm{O}$ nucleophile.

Coordinates for the TS along the deglycosylation of $5 \mathrm{hm}-\mathrm{dC}$ facilitated by the $\mathrm{HO}^{-} \ldots \mathrm{H}_{2} \mathrm{O}$ nucleophile.

Coordinates for the TS along the deglycosylation of $5 \mathrm{f}-\mathrm{dC}$ facilitated by the $\mathrm{HO}^{-} \ldots \mathrm{H}_{2} \mathrm{O}$ nucleophile.

Coordinates for the TS along the deglycosylation of 5ca-dC facilitated by the $\mathrm{HO}^{-} \cdots \mathrm{H}_{2} \mathrm{O}$ nucleophile.

Coordinates for the TS along the deglycosylation of dU facilitated by the $\mathrm{HO}^{-} \ldots \mathrm{H}_{2} \mathrm{O}$ nucleophile.

Coordinates for the TS along the deglycosylation of dT facilitated by the $\mathrm{HO}^{-} \ldots \mathrm{H}_{2} \mathrm{O}$ nucleophile.

Coordinates for the TS along the deglycosylation of 5hm-dU facilitated by the $\mathrm{HO}^{-} \cdots \mathrm{H}_{2} \mathrm{O}$ nucleophile.

Coordinates for the TS along the deglycosylation of $\mathrm{dC}$ facilitated by the $\mathrm{HO}^{-} \ldots \mathrm{H}_{2} \mathrm{O}$ nucleophile with a discrete water bound to the O2(N3) nucleobase site. S19

Coordinates for the TS along the deglycosylation of $\mathrm{dC}$ facilitated by the $\mathrm{HO}^{-} \ldots \mathrm{H}_{2} \mathrm{O}$ nucleophile with a discrete water bound to the N3(N4) nucleobase site. S19

Coordinates for the TS along the deglycosylation of $5 \mathrm{~m}-\mathrm{dC}$ facilitated by the $\mathrm{HO}^{-} \cdots \mathrm{H}_{2} \mathrm{O}$ nucleophile with a discrete water bound to the O2(N3) nucleobase site. S20

Coordinates for the TS along the deglycosylation of $5 \mathrm{~m}-\mathrm{dC}$ facilitated by the $\mathrm{HO}^{-} \ldots \mathrm{H}_{2} \mathrm{O}$ nucleophile with a discrete water bound to the N3(N4) nucleobase site.

Coordinates for the TS along the deglycosylation of $5 \mathrm{ca}-\mathrm{dC}$ facilitated by the $\mathrm{HO}^{-} \cdots \mathrm{H}_{2} \mathrm{O}$ nucleophile with a discrete water bound to the O2(N3) nucleobase site.

Coordinates for the TS along the deglycosylation of $5 \mathrm{ca}-\mathrm{dC}$ facilitated by the $\mathrm{HO}^{-} \cdots \mathrm{H}_{2} \mathrm{O}$ nucleophile with a discrete water bound to the N3(N4) nucleobase site.

Coordinates for the TS along the deglycosylation of dT facilitated by the $\mathrm{HO}^{-} \ldots \mathrm{H}_{2} \mathrm{O}$ nucleophile with a discrete water bound to the 02(N3) nucleobase site.

Coordinates for the TS along the deglycosylation of dT facilitated by the $\mathrm{HO}^{-} \ldots \mathrm{H}_{2} \mathrm{O}$ nucleophile with a discrete water bound to the 04(N3) nucleobase site. S25

Coordinates for the TS along the deglycosylation of 5hm-dU facilitated by the $\mathrm{HO}^{-} \cdots \mathrm{H}_{2} \mathrm{O}$ nucleophile with a discrete water bound to the 02(N3) nucleobase site. S26

Coordinates for the TS along the deglycosylation of $5 \mathrm{hm}$-dU facilitated by the $\mathrm{HO}^{-} \cdots \mathrm{H}_{2} \mathrm{O}$ nucleophile with a discrete water bound to the 04(N3) nucleobase site.

Full citation for references 17,27 , and 60 . S28 
Table S1. Calculated Gibbs Energy Barriers $\left(\Delta^{\ddagger} G\right)$ and Reaction Energies $\left(\Delta G_{\mathrm{rxn}}, \mathrm{kJ} / \mathrm{mol}\right)$ for the Hydrolytic Deglycosylation of the $5 \mathrm{ca}-\mathrm{dC}$ Nucleoside Facilitated by the $\mathrm{HO}^{-}$Nucleophile $^{a}$ with Different Conformations of the C5 Substituent.

\begin{tabular}{llll}
\hline nucleoside & orientation $^{\text {b }}$ & $\Delta^{\ddagger} G$ & $\Delta G_{\text {rxn }}$ \\
\hline $5 c a-d C$ & $\mathrm{C} 4$ & 111.1 & -86.9 \\
$5 \mathrm{ca}-\mathrm{dC}$ & $\mathrm{O5}^{\prime}$ & 116.3 & -75.7 \\
$5 \mathrm{ca}-\mathrm{dC}$ & $\mathrm{N} 4$ & 118.4 & -81.3 \\
$5 \mathrm{ca}-\mathrm{dC}$ & $\mathrm{C} 6$ & 120.2 & -76.2 \\
\hline${ }^{a} \mathrm{SMD}-\mathrm{B} 3 \mathrm{LYP} / 6-311++\mathrm{G}(2 \mathrm{df}, 2 \mathrm{p})$ single-point calculations were performed on IEF-PCM-B3LYP/6-31G(d) \\
geometries, with reported energies including scaled (0.9813) zero-point energy corrections and thermal \\
Gibbs energy corrections. ${ }^{b}$ Denotes the direction that the carboxylic acid proton is pointed with respect \\
to the nucleotide. See Figure S1.
\end{tabular}



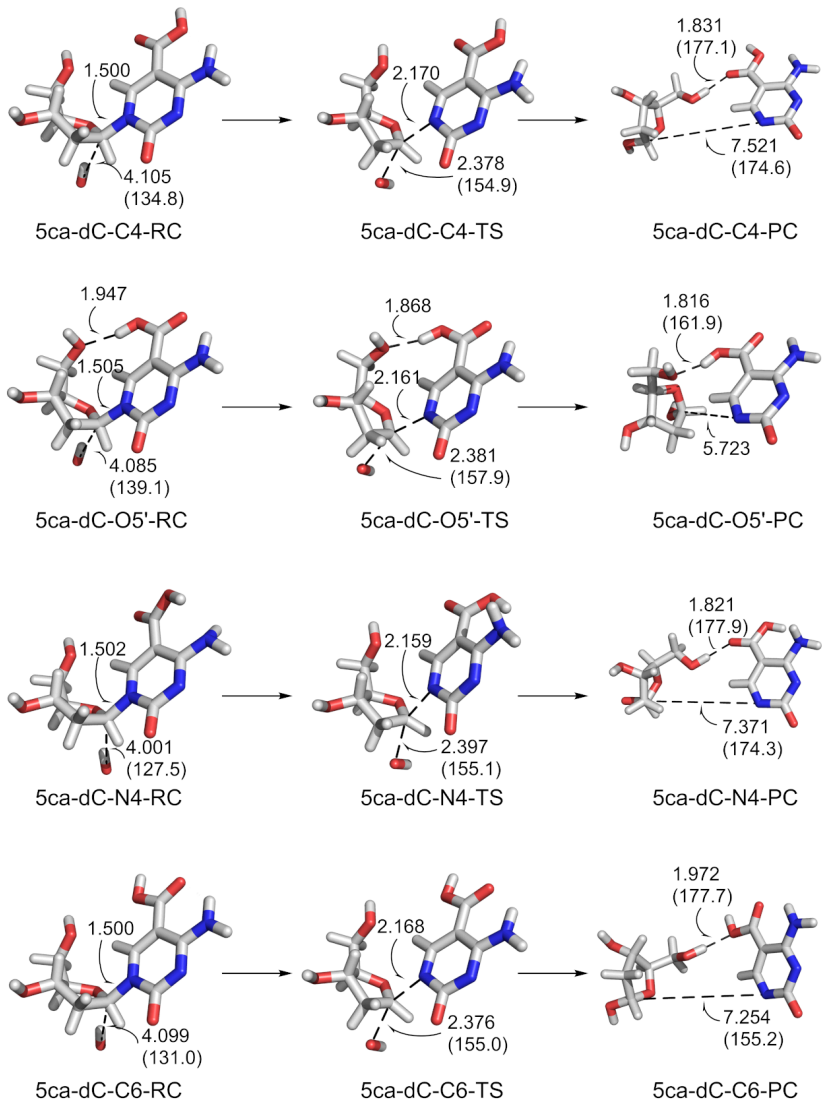

Figure S1. Stationary points (reactant complexes (RC), transition states (TS), and product complexes (PC)) along the deglycosylation of the $5 \mathrm{ca}-\mathrm{dC}$ nucleoside with different $\mathrm{C} 5$ substituent conformations facilitated by the $\mathrm{HO}^{-}$nucleophile.

Coordinates for the TS along the deglycosylation of $\mathrm{dC}$ facilitated by the $\mathrm{HO}^{-}$nucleophile.

$\begin{array}{llll}\mathrm{C} & -2.66181200 & 2.05720100 & -0.57952200 \\ \mathrm{H} & -2.84201300 & 2.20749100 & -1.65225800 \\ \mathrm{H} & -3.54554900 & 2.41063500 & -0.03078900 \\ \mathrm{C} & -2.52407100 & 0.57644500 & -0.30358500 \\ \mathrm{O} & -1.50359200 & -0.01723700 & -1.16933400 \\ \mathrm{C} & -0.97484500 & -1.07558600 & -0.57271700 \\ \mathrm{H} & -0.41389300 & -1.76954900 & -1.16361300 \\ \mathrm{~N} & 1.00649800 & -0.29770700 & -0.12331200 \\ \mathrm{C} & 1.35003800 & 0.97217400 & -0.36655900 \\ \mathrm{H} & 0.53012700 & 1.64547100 & -0.61265700 \\ \mathrm{C} & 2.65417100 & 1.42943600 & -0.29888800 \\ \mathrm{C} & 3.62623900 & 0.45747900 & 0.04666700 \\ \mathrm{~N} & 3.31436900 & -0.81798000 & 0.28273700 \\ \mathrm{C} & 1.99802800 & -1.21654900 & 0.18423300 \\ \mathrm{O} & 1.68210900 & -2.42235300 & 0.37646200 \\ \mathrm{C} & -2.11264100 & 0.13465300 & 1.10806700\end{array}$




$\begin{array}{lrrr}\mathrm{C} & -1.45374800 & -1.23333700 & 0.84212100 \\ \mathrm{H} & -0.64107400 & -1.48330400 & 1.52335700 \\ \mathrm{H} & -2.21336900 & -2.01847900 & 0.86209300 \\ \mathrm{H} & -1.39197900 & 0.84302000 & 1.52886600 \\ \mathrm{H} & -3.47201000 & 0.09064000 & -0.56312800 \\ \mathrm{O} & -2.52193900 & -2.62910000 & -1.29918400 \\ \mathrm{H} & -2.48633300 & -2.58562700 & -2.27101900 \\ \mathrm{O} & -3.28569000 & 0.08962600 & 1.91816100 \\ \mathrm{O} & -1.49001400 & 2.75703400 & -0.15929200 \\ \mathrm{H} & -3.01855300 & 0.00172400 & 2.84697500 \\ \mathrm{H} & -1.61739200 & 3.70270300 & -0.33433900 \\ \mathrm{H} & 2.91335500 & 2.46384200 & -0.49742400 \\ \mathrm{~N} & 4.96383500 & 0.79357200 & 0.10001700 \\ \mathrm{H} & 5.55792200 & 0.14309800 & 0.60100100 \\ \mathrm{H} & 5.19269000 & 1.76788100 & 0.25344000\end{array}$

Coordinates for the TS along the deglycosylation of $5 \mathrm{~m}-\mathrm{dC}$ facilitated by the $\mathrm{HO}^{-}$nucleophile.

$\begin{array}{lrrr}\mathrm{C} & -2.56149700 & 2.25759400 & -0.45253600 \\ \mathrm{H} & -2.68928500 & 2.50197900 & -1.51550700 \\ \mathrm{H} & -3.40804700 & 2.68608500 & 0.10151600 \\ \mathrm{C} & -2.62605200 & 0.75620800 & -0.28005900 \\ \mathrm{O} & -1.66751200 & 0.09289400 & -1.16647900 \\ \mathrm{C} & -1.29218800 & -1.05984700 & -0.63427900 \\ \mathrm{H} & -0.79515000 & -1.77321900 & -1.25809500 \\ \mathrm{~N} & 0.76361700 & -0.56614400 & -0.07408400 \\ \mathrm{C} & 1.27875600 & 0.65320900 & -0.27571300 \\ \mathrm{H} & 0.55625000 & 1.43400600 & -0.51320900 \\ \mathrm{C} & 2.62796300 & 0.95507300 & -0.18116200 \\ \mathrm{C} & 3.44900100 & -0.16105100 & 0.14398100 \\ \mathrm{~N} & 2.97010500 & -1.39230000 & 0.33181300 \\ \mathrm{C} & 1.61632400 & -1.61471200 & 0.21695200 \\ \mathrm{O} & 1.14406100 & -2.77649000 & 0.37228600 \\ \mathrm{C} & -2.31905900 & 0.16690200 & 1.10412400 \\ \mathrm{C} & -1.83303300 & -1.25306200 & 0.75271000 \\ \mathrm{H} & -1.08064400 & -1.65701700 & 1.42922600 \\ \mathrm{H} & -2.68812400 & -1.93127600 & 0.69626200 \\ \mathrm{H} & -1.52784900 & 0.74433800 & 1.59264500 \\ \mathrm{H} & -3.62190800 & 0.42120100 & -0.59292600 \\ \mathrm{O} & -2.97553700 & -2.37717100 & -1.51778000 \\ \mathrm{H} & -2.88812600 & -2.29671700 & -2.48397900 \\ \mathrm{O} & -3.51379500 & 0.21798800 & 1.88182900 \\ \mathrm{O} & -1.32196100 & 2.76699400 & 0.04006600 \\ \mathrm{C} & 3.17350200 & 2.34414200 & -0.39387800 \\ \mathrm{H} & 3.70221100 & 2.72325200 & 0.49211100 \\ \mathrm{H} & 3.87975200 & 2.39494200 & -1.23576400 \\ \mathrm{H} & 2.35968800 & 3.04351100 & -0.61418700 \\ \mathrm{H} & -3.28916800 & 0.03853600 & 2.80876500 \\ \mathrm{H} & -1.31389600 & 3.72901200 & -0.08447300\end{array}$




$\begin{array}{rrrr}\mathrm{N} & 4.80796000 & 0.00641500 & 0.33025500 \\ \mathrm{H} & 5.35387100 & -0.84721600 & 0.30686100 \\ \mathrm{H} & 5.25429400 & 0.79233300 & -0.12536000\end{array}$

Coordinates for the TS along the deglycosylation of $5 \mathrm{hm}-\mathrm{dC}$ facilitated by the $\mathrm{HO}^{-}$nucleophile.

$\mathrm{C}$

$\mathrm{H} \quad-2.56797700 \quad 2.74254600 \quad-1.42588600$

H $\quad-3.33747200 \quad 2.93860000 \quad 0.16389300$

C $\quad-2.74579400 \quad 0.94774800 \quad-0.27299500$

O $\quad-1.82724200 \quad 0.23319700-1.16558000$

C $\quad-1.61839300-0.98639900-0.69299300$

$\mathrm{H} \quad-1.21404300-1.72957900-1.34882100$

N $\quad 0.45495200-0.78360100-0.08149400$

$\begin{array}{llll}\text { C } & 1.09353600 & 0.38776600 & -0.13693700\end{array}$

$\mathrm{H} \quad 0.46921800 \quad 1.25828800-0.34036300$

$\begin{array}{llll}\text { C } & 2.45672000 & 0.53415200 & 0.07202100\end{array}$

$\begin{array}{llll}\text { C } & 3.15258700 & -0.68612700 & 0.32127000\end{array}$

$\begin{array}{lllll}\mathrm{N} & 2.54406400 & -1.87001600 & 0.36890900\end{array}$

C $\quad \begin{array}{llll}\text { C } & 1.18117200 & -1.93698500 & 0.17203500\end{array}$

$\begin{array}{lllll}0 & 0.58508400 & -3.04655700 & 0.20846200\end{array}$

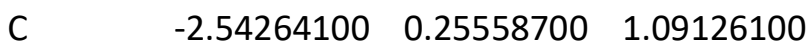

C $\quad-2.21019800-1.18370200 \quad 0.67328300$

$\mathrm{H} \quad-1.52668200 \quad-1.69930200 \quad 1.34677200$

H $\quad-3.13327500 \quad-1.75972200 \quad 0.57088500$

H $\quad-1.70573000 \quad 0.71417900 \quad 1.62637600$

H $\quad-3.75727800 \quad 0.72589100 \quad-0.63149700$

O $\quad-3.47362200-1.97204500-1.66071700$

H $\quad-3.36349100 \quad-1.84665400 \quad-2.61970100$

$\begin{array}{llll}0 & -3.71647700 & 0.28099800 & 1.90110000\end{array}$

$\begin{array}{lllll}0 & -1.25402000 & 2.78847600 & 0.19714300\end{array}$

$\begin{array}{llll}\text { C } & 3.13708400 & 1.87061100 & 0.07803700\end{array}$

$\begin{array}{llll}\mathrm{H} & 3.62598300 & 2.07385800 & 1.03785700\end{array}$

H $\quad 2.40021800 \quad 2.66485600 \quad-0.09775600$

$\mathrm{H} \quad-3.73634800 \quad 1.11020700 \quad 2.40481700$

$\begin{array}{llll}\mathrm{H} & -1.13720400 & 3.74871000 & 0.12433000\end{array}$

$\mathrm{N} \quad 4.50991500-0.65893300 \quad 0.56009300$

$\mathrm{H} \quad 4.97289100 \quad-1.55951800 \quad 0.51294400$

H $\quad 5.02210300 \quad 0.10207400 \quad 0.12615200$

$\begin{array}{lllll}\mathrm{O} & 4.21762700 & 1.97487200 & -0.88296100\end{array}$

H $\quad 3.85457100 \quad 1.77738700 \quad-1.76304700$

Coordinates for the TS along the deglycosylation of $5 \mathrm{f}-\mathrm{dC}$ facilitated by the $\mathrm{HO}^{-}$nucleophile.

$\begin{array}{llrr}\text { C } & -2.42995900 & 2.45513800 & -0.37470200 \\ \text { H } & -2.52740500 & 2.75247300 & -1.42712100 \\ \text { H } & -3.20470700 & 2.97771900 & 0.20285000 \\ \text { C } & -2.69990400 & 0.97217700 & -0.25096500 \\ \text { O } & -1.83291400 & 0.21559600 & -1.16164800 \\ \text { C } & -1.58823600 & -0.98051100 & -0.65922300\end{array}$




$\begin{array}{lrrr}\text { H } & -1.21688300 & -1.73964600 & -1.31777400 \\ \text { N } & 0.49474500 & -0.74252600 & -0.12015800 \\ \mathrm{C} & 1.12103200 & 0.40012000 & -0.30736400 \\ \mathrm{H} & 0.49869500 & 1.25656000 & -0.57000600 \\ \mathrm{C} & 2.50994500 & 0.55945200 & -0.16639300 \\ \mathrm{C} & 3.22481100 & -0.63895000 & 0.20519600 \\ \mathrm{~N} & 2.60614200 & -1.80608000 & 0.37910400 \\ \mathrm{C} & 1.24594000 & -1.88631000 & 0.20510000 \\ \mathrm{O} & 0.64389500 & -2.97400000 & 0.33088600 \\ \mathrm{C} & -2.47886900 & 0.30343000 & 1.11378900 \\ \mathrm{C} & -2.15630600 & -1.15137800 & 0.71794300 \\ \mathrm{H} & -1.46641300 & -1.66340700 & 1.38828300 \\ \mathrm{H} & -3.08227000 & -1.72530900 & 0.62796700 \\ \mathrm{H} & -1.63235100 & 0.76820100 & 1.62902200 \\ \mathrm{H} & -3.72833300 & 0.78098300 & -0.57732000 \\ \mathrm{O} & -3.47297500 & -2.10199300 & -1.57527700 \\ \mathrm{H} & -3.42403600 & -2.01919500 & -2.54393400 \\ \mathrm{O} & -3.66860300 & 0.46576100 & 1.88231600 \\ \mathrm{O} & -1.12770800 & 2.76475100 & 0.12224600 \\ \mathrm{C} & 3.11175600 & 1.84115500 & -0.38689400 \\ \mathrm{H} & -3.47309400 & 0.25224700 & 2.80867600 \\ \mathrm{H} & -0.98516500 & 3.72092200 & 0.04211000 \\ \mathrm{~N} & 4.56152200 & -0.58935000 & 0.38629900 \\ \mathrm{H} & 5.06586200 & -1.43379200 & 0.62029100 \\ \mathrm{H} & 5.05053500 & 0.28635300 & 0.23291200 \\ \mathrm{O} & 4.32370600 & 2.11248500 & -0.29143200 \\ \mathrm{H} & 2.40423600 & 2.64484300 & -0.66832100\end{array}$

Coordinates for the TS along the deglycosylation of 5ca-dC (C4) facilitated by the $\mathrm{HO}^{-}$nucleophile.

$\begin{array}{lrrr}\mathrm{C} & -2.41074300 & 2.53111600 & -0.20504900 \\ \mathrm{H} & -2.43494700 & 2.90588300 & -1.23669900 \\ \mathrm{H} & -3.17284300 & 3.07091800 & 0.37410000 \\ \mathrm{C} & -2.79315700 & 1.06819800 & -0.20091300 \\ \mathrm{O} & -1.95036700 & 0.31617900 & -1.13803700 \\ \mathrm{C} & -1.79754100 & -0.92302800 & -0.71237400 \\ \mathrm{H} & -1.45132700 & -1.65928400 & -1.40942400 \\ \mathrm{~N} & 0.28258500 & -0.87195100 & -0.09801200 \\ \mathrm{C} & 1.02445300 & 0.20704800 & -0.24239100 \\ \mathrm{H} & 0.49847800 & 1.12953600 & -0.48113000 \\ \mathrm{C} & 2.42100900 & 0.23064200 & -0.08798800 \\ \mathrm{C} & 3.00909000 & -1.03838300 & 0.26464700 \\ \mathrm{~N} & 2.26656100 & -2.14207800 & 0.38577600 \\ \mathrm{C} & 0.91249700 & -2.09251100 & 0.18404400 \\ \mathrm{O} & 0.21100300 & -3.12675100 & 0.24789500 \\ \mathrm{C} & -2.67030900 & 0.29093300 & 1.11795600 \\ \mathrm{C} & -2.42433700 & -1.15022900 & 0.63038700 \\ \mathrm{H} & -1.79215600 & -1.75157400 & 1.28295700\end{array}$




$\begin{array}{lrrr}\mathrm{H} & -3.37999200 & -1.65636500 & 0.46964300 \\ \mathrm{H} & -1.81806200 & 0.66151500 & 1.69599800 \\ \mathrm{H} & -3.82054600 & 0.97954200 & -0.57180900 \\ \mathrm{O} & -3.70763700 & -1.86251500 & -1.77074900 \\ \mathrm{H} & -3.61099100 & -1.74206500 & -2.73186600 \\ \mathrm{O} & -3.87803800 & 0.47705300 & 1.85304800 \\ \mathrm{O} & -1.11596700 & 2.70332200 & 0.36963300 \\ \mathrm{C} & 3.10329100 & 1.49692700 & -0.30029500 \\ \mathrm{H} & -3.73403200 & 0.18706400 & 2.76797000 \\ \mathrm{H} & -0.87245400 & 3.64028100 & 0.30869400 \\ \mathrm{O} & 4.45971600 & 1.44954700 & -0.12384200 \\ \mathrm{O} & 2.56657800 & 2.55715900 & -0.61748200 \\ \mathrm{~N} & 4.33415700 & -1.18518000 & 0.50985700 \\ \mathrm{H} & 4.68732400 & -2.12099400 & 0.65647800 \\ \mathrm{H} & 4.98267900 & -0.43051800 & 0.33885000 \\ \mathrm{H} & 4.80792200 & 2.34429300 & -0.29896600\end{array}$

Coordinates for the TS along the deglycosylation of 5ca-dC (O5') facilitated by the $\mathrm{HO}^{-}$nucleophile.

$\mathrm{C}$

$\mathrm{H}$

$\mathrm{H}$

C

O

C

$\mathrm{H}$

$\mathrm{N}$

C

$\mathrm{H}$

C

C

$\mathrm{N}$

C

O

C

C

$\mathrm{H}$

$\mathrm{H}$

$\mathrm{H}$

$\mathrm{H}$

$\mathrm{O}$

$\mathrm{H}$

O

O

C

$\mathrm{H}$

$\mathrm{H}$

$\mathrm{O}$

O $\begin{array}{lll}-1.78700700 & 2.58444000 & -0.04367100\end{array}$

$\begin{array}{lll}-1.78525200 & 2.99307400 & -1.05785800\end{array}$

$\begin{array}{lll}-2.39012700 & 3.24575500 & 0.58729800\end{array}$

$\begin{array}{llll}-2.44989600 & 1.21419800 & -0.06191600\end{array}$

$\begin{array}{llll}-1.90338800 & 0.39830300 & -1.15342600\end{array}$

$\begin{array}{lll}-1.91948200 & -0.88045400 & -0.82033500\end{array}$

$-1.78882600-1.60183600-1.60154600$

$0.19037800-1.11364300-0.41143600$

$0.90115700-0.01872400-0.58438400$

$\begin{array}{llll}0.36388600 & 0.80294900 & -1.04763900\end{array}$

$\begin{array}{llll}2.24596500 & 0.11892600 & -0.23186500\end{array}$

$\begin{array}{llll}2.85189000 & -1.08973600 & 0.27209700\end{array}$

$\begin{array}{llll}2.14804100 & -2.20984600 & 0.44873600\end{array}$

$\begin{array}{lll}0.81002500 & -2.24198400 & 0.14352600\end{array}$

$\begin{array}{llll}0.12590600 & -3.27079300 & 0.33695600\end{array}$

$\begin{array}{lll}-2.33311300 & 0.31430600 & 1.17753200\end{array}$

$\begin{array}{lll}-2.42353400 & -1.10182100 & 0.57265600\end{array}$

$\begin{array}{lll}-1.84870800 & -1.86157800 & 1.10274000\end{array}$

$\begin{array}{lll}-3.47059500 & -1.40788600 & 0.49931700\end{array}$

$\begin{array}{lll}-1.36495100 & 0.45975100 & 1.66944500\end{array}$

$\begin{array}{llll}-3.51119200 & 1.35902200 & -0.29595000\end{array}$

$-4.07624500-1.42414700-1.66990600$

$\begin{array}{lll}-4.08733400 & -1.29634000 & -2.63491800\end{array}$

$\begin{array}{llll}-3.38901700 & 0.65745400 & 2.06968900\end{array}$

$\begin{array}{lll}-0.42208800 & 2.59346800 & 0.40158100\end{array}$

$\begin{array}{llll}2.96507600 & 1.37988200 & -0.37429900\end{array}$

$\begin{array}{lll}-3.22349500 & 0.23247000 & 2.92663300\end{array}$

$\begin{array}{lll}-0.38743800 & 2.70978900 & 1.36403600\end{array}$

$\begin{array}{llll}2.24699600 & 2.51931200 & -0.55039800\end{array}$

$\begin{array}{llll}4.19439400 & 1.48401500 & -0.34406300\end{array}$ 


\begin{tabular}{|c|c|c|c|}
\hline $\mathrm{N}$ & 4.16109900 & -1.11538500 & 0.61336000 \\
\hline $\mathrm{H}$ & 4.57536700 & -1.99943200 & 0.87594000 \\
\hline $\mathrm{H}$ & 4.75021200 & -0.33134800 & 0.35815700 \\
\hline $\mathrm{H}$ & 1.29287100 & 2.43263300 & -0.31977200 \\
\hline
\end{tabular}

Coordinates for the TS along the deglycosylation of $5 \mathrm{ca}-\mathrm{dC}(\mathrm{N} 4)$ facilitated by the $\mathrm{HO}^{-}$nucleophile.

$\begin{array}{lrrr}\mathrm{C} & -2.42243600 & 2.52890300 & -0.14320700 \\ \mathrm{H} & -2.45078100 & 2.93252900 & -1.16378700 \\ \mathrm{H} & -3.18638700 & 3.04768200 & 0.45238200 \\ \mathrm{C} & -2.79719700 & 1.06444900 & -0.17944800 \\ \mathrm{O} & -1.94329600 & 0.34109900 & -1.13045500 \\ \mathrm{C} & -1.78001100 & -0.90552900 & -0.73381700 \\ \mathrm{H} & -1.43334000 & -1.62319400 & -1.45058300 \\ \mathrm{~N} & 0.28151600 & -0.85050500 & -0.09760700 \\ \mathrm{C} & 1.00543600 & 0.24749000 & -0.20926600 \\ \mathrm{H} & 0.47070200 & 1.16131500 & -0.46026300 \\ \mathrm{C} & 2.39672400 & 0.27590400 & -0.02512100 \\ \mathrm{C} & 2.96314600 & -0.96779100 & 0.38389500 \\ \mathrm{~N} & 2.26737200 & -2.08659300 & 0.45657000 \\ \mathrm{C} & 0.91964400 & -2.06966800 & 0.14609300 \\ \mathrm{O} & 0.25874200 & -3.12626700 & 0.10540400 \\ \mathrm{C} & -2.68032800 & 0.25266800 & 1.11911100 \\ \mathrm{C} & -2.42125200 & -1.17314000 & 0.59444300 \\ \mathrm{H} & -1.79726500 & -1.79046000 & 1.24029300 \\ \mathrm{H} & -3.37180900 & -1.67902500 & 0.40439600 \\ \mathrm{H} & -1.83517100 & 0.61222800 & 1.71441500 \\ \mathrm{H} & -3.82094800 & 0.98013000 & -0.56100200 \\ \mathrm{O} & -3.69055500 & -1.84593100 & -1.83523700 \\ \mathrm{H} & -3.59943800 & -1.71827000 & -2.79594500 \\ \mathrm{O} & -3.89510200 & 0.41088700 & 1.84813200 \\ \mathrm{O} & -1.12722600 & 2.69183000 & 0.43328700 \\ \mathrm{C} & 3.13819400 & 1.49850200 & -0.36890000 \\ \mathrm{H} & -3.75640400 & 0.10017500 & 2.75709600 \\ \mathrm{H} & -0.89335600 & 3.63293300 & 0.40858100 \\ \mathrm{O} & 4.48145100 & 1.39528300 & -0.53876100 \\ \mathrm{O} & 2.61145800 & 2.59091300 & -0.55172800 \\ \mathrm{~N} & 4.33402400 & -1.04325800 & 0.69002800 \\ \mathrm{H} & 4.61904100 & -0.42981600 & 1.45270400 \\ \mathrm{H} & 4.60443000 & -1.99970900 & 0.90994600 \\ \mathrm{H} & 4.77505800 & 0.46274000 & -0.39901100\end{array}$

Coordinates for the TS along the deglycosylation of $5 \mathrm{ca}-\mathrm{dC}(\mathrm{C} 6)$ facilitated by the $\mathrm{HO}^{-}$nucleophile.

$\begin{array}{llll}\mathrm{C} & -2.40509100 & 2.51531700 & -0.24327700 \\ \mathrm{H} & -2.43649900 & 2.87356800 & -1.28053700 \\ \mathrm{H} & -3.16431100 & 3.06290800 & 0.33227200 \\ \mathrm{C} & -2.78325300 & 1.05159600 & -0.21311000 \\ \mathrm{O} & -1.94021500 & 0.28731900 & -1.13950300 \\ \mathrm{C} & -1.78120500 & -0.94428800 & -0.69334400\end{array}$




$\begin{array}{lrrr}\mathrm{H} & -1.43508800 & -1.69156500 & -1.37853400 \\ \mathrm{~N} & 0.30005600 & -0.87804300 & -0.08964800 \\ \mathrm{C} & 1.03513200 & 0.20876000 & -0.22255800 \\ \mathrm{H} & 0.49712500 & 1.12821300 & -0.44440000 \\ \mathrm{C} & 2.43158000 & 0.22534200 & -0.07272600 \\ \mathrm{C} & 3.02339400 & -1.04884100 & 0.25223300 \\ \mathrm{~N} & 2.28928800 & -2.15761300 & 0.36515400 \\ \mathrm{C} & 0.93290200 & -2.10197600 & 0.17538400 \\ \mathrm{O} & 0.22949600 & -3.13516800 & 0.23466700 \\ \mathrm{C} & -2.65341700 & 0.29774600 & 1.11877300 \\ \mathrm{C} & -2.40232200 & -1.15067100 & 0.65558500 \\ \mathrm{H} & -1.76504800 & -1.73692000 & 1.31691700 \\ \mathrm{H} & -3.35625800 & -1.66383000 & 0.50785300 \\ \mathrm{H} & -1.80136000 & 0.68215700 & 1.68807000 \\ \mathrm{H} & -3.81146100 & 0.95296200 & -0.57915100 \\ \mathrm{O} & -3.69301500 & -1.90335300 & -1.72654300 \\ \mathrm{H} & -3.60292900 & -1.78736900 & -2.68881900 \\ \mathrm{O} & -3.85994100 & 0.49128700 & 1.85394100 \\ \mathrm{O} & -1.10673200 & 2.69860200 & 0.32005400 \\ \mathrm{C} & 3.21279500 & 1.43285000 & -0.23880000 \\ \mathrm{H} & -3.71191000 & 0.21872600 & 2.77355800 \\ \mathrm{H} & -0.86353300 & 3.63423700 & 0.24078900 \\ \mathrm{O} & 2.47778200 & 2.53384700 & -0.55790500 \\ \mathrm{O} & 4.44059700 & 1.52386200 & -0.11988500 \\ \mathrm{~N} & 4.35384200 & -1.16378300 & 0.46452600 \\ \mathrm{H} & 4.74577900 & -2.08139100 & 0.62556000 \\ \mathrm{H} & 4.95424400 & -0.35970200 & 0.32583800 \\ \mathrm{H} & 3.10208000 & 3.27791800 & -0.64719900\end{array}$

Coordinates for the TS along the deglycosylation of dU facilitated by the $\mathrm{HO}^{-}$nucleophile.

$\begin{array}{lrrr}\mathrm{C} & -2.61122700 & 2.08965600 & -0.53385500 \\ \mathrm{H} & -2.77277300 & 2.27632800 & -1.60375100 \\ \mathrm{H} & -3.49664100 & 2.44155700 & 0.01314700 \\ \mathrm{C} & -2.50345800 & 0.59924500 & -0.30169200 \\ \mathrm{O} & -1.47686700 & 0.01469200 & -1.17214200 \\ \mathrm{C} & -0.94900700 & -1.04754000 & -0.59279900 \\ \mathrm{H} & -0.40929800 & -1.74133300 & -1.20544900 \\ \mathrm{~N} & 1.00797700 & -0.28064400 & -0.10073400 \\ \mathrm{C} & 1.37406200 & 0.98627800 & -0.39540400 \\ \mathrm{H} & 0.55101800 & 1.64438300 & -0.66756400 \\ \mathrm{C} & 2.66284000 & 1.46758700 & -0.35153700 \\ \mathrm{C} & 3.72246900 & 0.57709000 & 0.02459900 \\ \mathrm{O} & 4.93759100 & 0.84880800 & 0.11597000 \\ \mathrm{~N} & 3.28111100 & -0.71968600 & 0.30390700 \\ \mathrm{H} & 3.98885400 & -1.40047600 & 0.56299800 \\ \mathrm{C} & 1.96549800 & -1.18863700 & 0.23978100 \\ \mathrm{O} & 1.72755400 & -2.38656400 & 0.49048400 \\ \mathrm{C} & -2.12197000 & 0.10633300 & 1.10153000\end{array}$




$\begin{array}{lrrr}\mathrm{C} & -1.46561300 & -1.25523100 & 0.80015000 \\ \mathrm{H} & -0.67856200 & -1.54451600 & 1.49596300 \\ \mathrm{H} & -2.23125000 & -2.03441900 & 0.75710600 \\ \mathrm{H} & -1.40561200 & 0.79468000 & 1.56092900 \\ \mathrm{H} & -3.45267100 & 0.13604800 & -0.59429200 \\ \mathrm{O} & -2.49372700 & -2.65455300 & -1.40764800 \\ \mathrm{H} & -2.46477400 & -2.62147600 & -2.38014500 \\ \mathrm{O} & -3.31081300 & 0.03908200 & 1.88480900 \\ \mathrm{O} & -1.43264900 & 2.75157200 & -0.07450700 \\ \mathrm{H} & -3.06370700 & -0.06391800 & 2.81761500 \\ \mathrm{H} & -1.53428100 & 3.70415400 & -0.22779900 \\ \mathrm{H} & 2.89580000 & 2.49830500 & -0.59133900\end{array}$

Coordinates for the TS along the deglycosylation of dT facilitated by the $\mathrm{HO}^{-}$nucleophile.

$\begin{array}{lrrr}\mathrm{C} & -2.52494500 & 2.27174400 & -0.44542100 \\ \mathrm{H} & -2.63932900 & 2.52497800 & -1.50772900 \\ \mathrm{H} & -3.37275700 & 2.70449200 & 0.10331200 \\ \mathrm{C} & -2.60577700 & 0.77019300 & -0.28389500 \\ \mathrm{O} & -1.64061900 & 0.10402000 & -1.16542300 \\ \mathrm{C} & -1.26047200 & -1.04140900 & -0.63044000 \\ \mathrm{H} & -0.78799500 & -1.76380400 & -1.26464800 \\ \mathrm{~N} & 0.76921800 & -0.55486000 & -0.05437700 \\ \mathrm{C} & 1.30867600 & 0.67075600 & -0.26136100 \\ \mathrm{H} & 0.58070400 & 1.44674700 & -0.49386300 \\ \mathrm{C} & 2.64625000 & 0.99061000 & -0.18286500 \\ \mathrm{C} & 3.56038400 & -0.07609100 & 0.13952400 \\ \mathrm{O} & 4.80050300 & 0.02382500 & 0.25429500 \\ \mathrm{~N} & 2.94959500 & -1.31643600 & 0.32911000 \\ \mathrm{H} & 3.56071600 & -2.09842100 & 0.54476100 \\ \mathrm{C} & 1.58546500 & -1.60254100 & 0.22888500 \\ \mathrm{O} & 1.18800300 & -2.77475800 & 0.39949800 \\ \mathrm{C} & -2.31978100 & 0.16807400 & 1.09923000 \\ \mathrm{C} & -1.83190600 & -1.24937000 & 0.74105400 \\ \mathrm{H} & -1.10310000 & -1.66901100 & 1.43379100 \\ \mathrm{H} & -2.68824600 & -1.92231000 & 0.64581300 \\ \mathrm{H} & -1.53386200 & 0.73883000 & 1.60392900 \\ \mathrm{H} & -3.59884600 & 0.44394000 & -0.61389200 \\ \mathrm{O} & -2.95601700 & -2.41166100 & -1.55139200 \\ \mathrm{H} & -2.88412400 & -2.35720700 & -2.52066000 \\ \mathrm{O} & -3.52488500 & 0.21374000 & 1.85924800 \\ \mathrm{O} & -1.28508000 & 2.76271600 & 0.06378500 \\ \mathrm{C} & 3.17584000 & 2.38074900 & -0.41257700 \\ \mathrm{H} & 3.70117800 & 2.76676700 & 0.47107700 \\ \mathrm{H} & 3.89288600 & 2.41048100 & -1.24363600 \\ \mathrm{H} & 2.35836400 & 3.07082200 & -0.64722200 \\ \mathrm{H} & -3.31306800 & 0.03844900 & 2.79003300 \\ \mathrm{H} & -1.26185600 & 3.72497100 & -0.05709500 \\ & & & \\ & & & \end{array}$


Coordinates for the TS along the deglycosylation of $5 \mathrm{hm}$ - $\mathrm{dU}$ facilitated by the $\mathrm{HO}^{-}$nucleophile.

\begin{tabular}{llll}
$\mathrm{C}$ & 2.44344600 & 2.48666600 & 0.33200000 \\
$\mathrm{H}$ & 2.46479200 & 2.82145400 & 1.37741900 \\
$\mathrm{H}$ & 3.25514700 & 2.99389200 & -0.20735000 \\
$\mathrm{C}$ & 2.72989000 & 1.00263200 & 0.28091200 \\
$\mathrm{O}$ & 1.81784400 & 0.27037800 & 1.16851200 \\
$\mathrm{C}$ & 1.60551600 & -0.94224800 & 0.69536900 \\
$\mathrm{H}$ & 1.20758400 & -1.68107100 & 1.36167000 \\
$\mathrm{C}$ & 2.59407100 & 0.28106600 & -1.06784900 \\
$\mathrm{C}$ & 2.26128000 & -1.16045900 & -0.63544100 \\
$\mathrm{H}$ & 1.62304900 & -1.70737900 & -1.32893900 \\
$\mathrm{H}$ & 3.18537000 & -1.71818500 & -0.46055200 \\
$\mathrm{H}$ & 1.77489200 & 0.71668600 & -1.64848500 \\
$\mathrm{H}$ & 3.73881500 & 0.83444800 & 0.67403000 \\
$\mathrm{O}$ & 3.43503900 & -2.04192100 & 1.76704800 \\
$\mathrm{H}$ & 3.33159300 & -1.95240800 & 2.73083800 \\
$\mathrm{O}$ & 3.82512400 & 0.42651200 & -1.77158100 \\
$\mathrm{O}$ & 1.17910300 & 2.76954600 & -0.26738200 \\
$\mathrm{H}$ & 3.68591200 & 0.17681700 & -2.69902900 \\
$\mathrm{H}$ & 1.02168900 & 3.72563200 & -0.21962500 \\
$\mathrm{~N}$ & -0.44060600 & -0.74568600 & 0.02678500 \\
$\mathrm{C}$ & -1.12129900 & 0.41556400 & 0.14065700 \\
$\mathrm{H}$ & -0.50297700 & 1.28243700 & 0.37037800 \\
$\mathrm{C}$ & -2.47939600 & 0.56377600 & -0.03745200 \\
$\mathrm{C}$ & -3.24667200 & -0.61553500 & -0.33129600 \\
$\mathrm{~N}$ & -2.49920700 & -1.78247600 & -0.42465400 \\
$\mathrm{C}$ & -1.11553800 & -1.89686700 & -0.24381600 \\
$\mathrm{O}$ & -0.57624600 & -3.01653800 & -0.33119400 \\
$\mathrm{C}$ & -3.20349400 & 1.88009700 & 0.04241500 \\
$\mathrm{H}$ & -2.53316200 & 2.66447000 & 0.40432400 \\
$\mathrm{H}$ & -3.56862700 & 2.18180700 & -0.95143900 \\
$\mathrm{O}$ & -4.49126900 & -0.64779900 & -0.48095500 \\
$\mathrm{O}$ & -4.32009500 & 1.84302700 & 0.95164400 \\
$\mathrm{H}$ & -4.86421800 & 1.08869400 & 0.65216300 \\
$\mathrm{H}$ & -3.00398600 & -2.64394700 & -0.61205200 \\
& & & \\
\hline
\end{tabular}

Coordinates for the TS along the deglycosylation of $\mathrm{dC}$ facilitated by the $\mathrm{HO}^{-} \cdots \mathrm{H}_{2} \mathrm{O}$ nucleophile.

$\mathrm{H} \quad-2.29526400 \quad 2.52277900-1.63682800$

$\mathrm{H} \quad-2.83235100 \quad 2.95544800 \quad 0.00312900$

C $\quad-2.09930900 \quad 0.97889800 \quad-0.16883100$

$\begin{array}{llll}0 & -1.23638800 & 0.17497900 & -1.04722900\end{array}$

C $\quad-0.81083300-0.88766500-0.39925800$

$\mathrm{H} \quad-0.33290600-1.66991700-0.95255900$

N $\quad 1.34788900-0.32345600-0.10780400$

C $\quad 1.83682700 \quad 0.87299200-0.45228300$

H $\quad 1.09734300 \quad 1.62853900 \quad-0.71461900$

C $\quad 3.18987000 \quad 1.16595300 \quad-0.46704100$ 


$\begin{array}{lrrr}\text { C } & 4.04867500 & 0.10453700 & -0.08889100 \\ \text { N } & 3.59205600 & -1.10294000 & 0.24837600 \\ \text { C } & 2.23276000 & -1.33606800 & 0.22623200 \\ \text { O } & 1.78053100 & -2.47888100 & 0.51424800 \\ \text { C } & -1.63579300 & 0.59360300 & 1.24392500 \\ \text { C } & -1.19168600 & -0.87083400 & 1.05117200 \\ H & -0.36639000 & -1.18567800 & 1.68883600 \\ H & -2.04517600 & -1.53828800 & 1.19557900 \\ H & -0.79237800 & 1.22067000 & 1.54892500 \\ H & -3.11996900 & 0.61078600 & -0.32253700 \\ \text { O } & -2.73138500 & 0.77565400 & 2.13665400 \\ \text { O } & -0.76513700 & 2.98992100 & -0.29495700 \\ H & -2.39681200 & 0.75743200 & 3.04734900 \\ H & -0.76837000 & 3.92271900 & -0.56082900 \\ \text { O } & -4.88770600 & -1.21073900 & -0.63060900 \\ H & -5.53070200 & -1.93442400 & -0.57844900 \\ \text { O } & -2.55056100 & -2.29895800 & -0.94970800 \\ H & -2.43690400 & -2.45056800 & -1.90344400 \\ H & -3.96932800 & -1.66339100 & -0.77170800 \\ H & 3.56505800 & 2.14416700 & -0.74829900 \\ \mathrm{~N} & 5.41962500 & 0.27148400 & -0.10733000 \\ \mathrm{H} & 5.77240500 & 1.21731800 & -0.02777500 \\ \mathrm{H} & 5.94846500 & -0.41275600 & 0.42119200\end{array}$

Coordinates for the TS along the deglycosylation of $5 \mathrm{~m}-\mathrm{dC}$ facilitated by the $\mathrm{HO}^{-} \cdots \mathrm{H}_{2} \mathrm{O}$ nucleophile.

$\begin{array}{lrrr}\mathrm{C} & -2.03848800 & 2.50924900 & -0.38977400 \\ \mathrm{H} & -2.23974200 & 2.68979200 & -1.45399400 \\ \mathrm{H} & -2.79011000 & 3.05400800 & 0.19807000 \\ \mathrm{C} & -2.22196800 & 1.03785300 & -0.09517800 \\ \mathrm{O} & -1.40728400 & 0.22377700 & -1.00913500 \\ \mathrm{C} & -1.09363000 & -0.91438600 & -0.42858100 \\ \mathrm{H} & -0.66349300 & -1.69420300 & -1.02313200 \\ \mathrm{~N} & 1.09867200 & -0.56279700 & -0.04789100 \\ \mathrm{C} & 1.70866900 & 0.59656400 & -0.32448700 \\ \mathrm{H} & 1.04723200 & 1.43143600 & -0.55765500 \\ \mathrm{C} & 3.08315900 & 0.77946900 & -0.31468100 \\ \mathrm{C} & 3.81879800 & -0.39005800 & 0.02411300 \\ \mathrm{~N} & 3.24417200 & -1.56409400 & 0.29263100 \\ \mathrm{C} & 1.87225700 & -1.67086400 & 0.23991300 \\ \mathrm{O} & 1.31007500 & -2.78285600 & 0.45763400 \\ \mathrm{C} & -1.83203000 & 0.51762600 & 1.29638500 \\ \mathrm{C} & -1.51217300 & -0.96356700 & 1.01073500 \\ \mathrm{H} & -0.73576000 & -1.39450800 & 1.64167800 \\ \mathrm{H} & -2.42435800 & -1.56067900 & 1.08905600 \\ \mathrm{H} & -0.94535500 & 1.04500400 & 1.66117000 \\ \mathrm{H} & -3.26614600 & 0.77247200 & -0.29667200 \\ \mathrm{O} & -2.93119200 & 0.73533900 & 2.17706600 \\ \mathrm{O} & -0.71909400 & 2.92976000 & -0.04295700\end{array}$




$\begin{array}{lrrr}\mathrm{C} & 3.73692200 & 2.09926900 & -0.63546900 \\ \mathrm{H} & 4.30794500 & 2.50540900 & 0.21266900 \\ \mathrm{H} & 4.43270700 & 2.02236400 & -1.48287600 \\ \mathrm{H} & 2.97985700 & 2.84574700 & -0.89972000 \\ \mathrm{H} & -2.62287400 & 0.62881300 & 3.09099900 \\ \mathrm{H} & -0.63358800 & 3.87422500 & -0.24728600 \\ \mathrm{O} & -5.16454400 & -0.82420200 & -0.83269300 \\ \mathrm{H} & -5.87869500 & -1.47404800 & -0.92000400 \\ \mathrm{O} & -2.92860700 & -2.12076800 & -1.11570500 \\ \mathrm{H} & -2.78793600 & -2.22954800 & -2.07172700 \\ \mathrm{H} & -4.28830800 & -1.35581600 & -0.96107000 \\ \mathrm{~N} & 5.20124500 & -0.36460700 & 0.03278100 \\ \mathrm{H} & 5.65518300 & 0.52586900 & 0.19278200 \\ \mathrm{H} & 5.64087700 & -1.14116300 & 0.51352100\end{array}$

Coordinates for the TS along the deglycosylation of $5 \mathrm{hm}-\mathrm{dC}$ facilitated by the $\mathrm{HO}^{-} \ldots \mathrm{H}_{2} \mathrm{O}$ nucleophile.

$\begin{array}{lrrr}\mathrm{C} & 1.96025100 & 2.66536800 & 0.33184900 \\ \mathrm{H} & 1.87245000 & 2.97866800 & 1.38046700 \\ \mathrm{H} & 2.77481500 & 3.23970700 & -0.13056700 \\ \mathrm{C} & 2.35407900 & 1.20550600 & 0.28296300 \\ \mathrm{O} & 1.43015400 & 0.39998900 & 1.08391100 \\ \mathrm{C} & 1.31209600 & -0.80552100 & 0.56671000 \\ \mathrm{H} & 0.91038300 & -1.59053500 & 1.17487400 \\ \mathrm{~N} & -0.82769000 & -0.71215100 & -0.04334600 \\ \mathrm{C} & -1.55785400 & 0.40259200 & 0.03811700 \\ \mathrm{H} & -0.99653700 & 1.32496500 & 0.19250200 \\ \mathrm{C} & -2.93963900 & 0.43375900 & -0.08699500 \\ \mathrm{C} & -3.54640600 & -0.84400700 & -0.26970700 \\ \mathrm{~N} & -2.84617700 & -1.97523700 & -0.33963600 \\ \mathrm{C} & -1.47231200 & -1.92344700 & -0.23548100 \\ \mathrm{O} & -0.78818900 & -2.98075700 & -0.29978600 \\ \mathrm{C} & 2.38954900 & 0.51855500 & -1.09416900 \\ \mathrm{C} & 1.92494000 & -0.92525300 & -0.79908600 \\ \mathrm{H} & 1.20292400 & -1.30517600 & -1.52126700 \\ \mathrm{H} & 2.77804800 & -1.60388400 & -0.75381700 \\ \mathrm{H} & 1.70885300 & 1.01924600 & -1.78795100 \\ \mathrm{H} & 3.33491300 & 1.09725400 & 0.75773100 \\ \mathrm{O} & 3.73165900 & 0.62113400 & -1.58479700 \\ \mathrm{O} & 0.73177900 & 2.87091900 & -0.36531500 \\ \mathrm{C} & -3.72555000 & 1.71074200 & -0.07143700 \\ \mathrm{H} & -4.28795700 & 1.85431800 & -1.00131100 \\ \mathrm{H} & -3.04569700 & 2.56481200 & 0.04409600 \\ \mathrm{H} & 3.74139200 & 0.38368900 & -2.52632900 \\ \mathrm{H} & 0.51933400 & 3.81724100 & -0.34346700 \\ \mathrm{O} & 5.41732100 & -0.91892600 & 0.36700200 \\ \mathrm{H} & 5.06106900 & -0.38911000 & -0.36957500 \\ \mathrm{O} & 3.19005800 & -1.69266700 & 1.54790800 \\ \mathrm{H} & 3.13548500 & -1.37306800 & 2.46430800\end{array}$




$\begin{array}{llrl}\mathrm{H} & 4.58123000 & -1.21791400 & 0.87155200 \\ \mathrm{~N} & -4.91485100 & -0.93425800 & -0.41594700 \\ \mathrm{H} & -5.29417800 & -1.86943600 & -0.31941400 \\ \mathrm{H} & -5.45825200 & -0.21047900 & 0.04315300 \\ \mathrm{O} & -4.74958900 & 1.75017900 & 0.95455900 \\ \mathrm{H} & -4.31770100 & 1.60248900 & 1.81307000\end{array}$

Coordinates for the TS along the deglycosylation of $5 \mathrm{f}-\mathrm{dC}$ facilitated by the $\mathrm{HO}^{-} \cdots \mathrm{H}_{2} \mathrm{O}$ nucleophile.

$\begin{array}{lrrr}\mathrm{C} & -2.02735800 & 2.56971400 & -0.24568700 \\ \mathrm{H} & -2.21481600 & 2.82144100 & -1.29775800 \\ \mathrm{H} & -2.72160600 & 3.15231000 & 0.37529400 \\ \mathrm{C} & -2.34577700 & 1.10968500 & -0.02111100 \\ \mathrm{O} & -1.59637900 & 0.27024000 & -0.97562000 \\ \mathrm{C} & -1.35237600 & -0.90229700 & -0.44428200 \\ \mathrm{H} & -1.00514100 & -1.68852100 & -1.08453400 \\ \mathrm{~N} & 0.84368000 & -0.71606300 & -0.04864600 \\ \mathrm{C} & 1.50762900 & 0.39107000 & -0.30610100 \\ \mathrm{H} & 0.90932800 & 1.27094900 & -0.54780700 \\ \mathrm{C} & 2.90975000 & 0.49050300 & -0.26384100 \\ \mathrm{C} & 3.59488000 & -0.73094600 & 0.08701600 \\ \mathrm{~N} & 2.93829600 & -1.86367800 & 0.33386100 \\ \mathrm{C} & 1.56643200 & -1.88232200 & 0.25826100 \\ \mathrm{O} & 0.92749700 & -2.93793500 & 0.46086400 \\ \mathrm{C} & -2.01061500 & 0.48940200 & 1.34334900 \\ \mathrm{C} & -1.79991000 & -0.99477000 & 0.98140600 \\ \mathrm{H} & -1.07843300 & -1.52429600 & 1.60282500 \\ \mathrm{H} & -2.75792100 & -1.52200700 & 1.00186200 \\ \mathrm{H} & -1.09105300 & 0.92781300 & 1.74327600 \\ \mathrm{H} & -3.40579800 & 0.94315000 & -0.24272900 \\ \mathrm{O} & -3.10029400 & 0.74147800 & 2.22481500 \\ \mathrm{O} & -0.67208200 & 2.84398600 & 0.10946500 \\ \mathrm{H} & -2.80630700 & 0.58487500 & 3.13623300 \\ \mathrm{H} & -0.49815900 & 3.78677200 & -0.03866700 \\ \mathrm{O} & -5.27772500 & -0.36280700 & -1.05694100 \\ \mathrm{H} & -6.09523100 & -0.88392000 & -1.05731600 \\ \mathrm{O} & -3.30271400 & -2.02819800 & -1.22123700 \\ \mathrm{H} & -3.15163600 & -2.19684000 & -2.16680300 \\ \mathrm{H} & -4.50540800 & -1.05025000 & -1.14287800 \\ \mathrm{~N} & 4.94226800 & -0.73945300 & 0.17229600 \\ \mathrm{H} & 5.42387400 & -1.59963500 & 0.39657300 \\ \mathrm{H} & 5.45702300 & 0.11038700 & -0.03334400 \\ \mathrm{C} & 3.55094000 & 1.73811400 & -0.55403700 \\ \mathrm{H} & 2.86181400 & 2.56766300 & -0.80462500 \\ \mathrm{O} & 4.77771900 & 1.95643100 & -0.54784700\end{array}$

Coordinates for the TS along the deglycosylation of $5 \mathrm{ca}-\mathrm{dC}$ facilitated by the $\mathrm{HO}^{-} \cdots \mathrm{H}_{2} \mathrm{O}$ nucleophile.

C $\quad-2.05830500 \quad 2.58805400 \quad-0.05818000$

H $\quad-2.17389300 \quad 2.92288300 \quad-1.09732600$ 


$\begin{array}{llll}\mathrm{H} & -2.75328400 & 3.16521800 & 0.56739500 \\ \mathrm{C} & -2.46394000 & 1.13596700 & 0.04346000 \\ \mathrm{O} & -1.71734800 & 0.32865700 & -0.93931000 \\ \mathrm{C} & -1.54811100 & -0.88778600 & -0.48315100 \\ \mathrm{H} & -1.21421300 & -1.64525700 & -1.16395200 \\ \mathrm{C} & -2.21908500 & 0.40536300 & 1.37254200 \\ \mathrm{C} & -2.05605400 & -1.05783700 & 0.91482200 \\ \mathrm{H} & -1.38225600 & -1.66054600 & 1.52277800 \\ \mathrm{H} & -3.03542000 & -1.54200200 & 0.86262300 \\ \mathrm{H} & -1.30106200 & 0.77275500 & 1.84106100 \\ \mathrm{H} & -3.52041600 & 1.04287100 & -0.23088200 \\ \mathrm{O} & -3.33647800 & 0.64516100 & 2.22302500 \\ \mathrm{O} & -0.71221200 & 2.75477900 & 0.38484000 \\ \mathrm{H} & -3.09109600 & 0.41319000 & 3.13272500 \\ \mathrm{H} & -0.46580300 & 3.68741300 & 0.28378300 \\ \mathrm{O} & -5.45585100 & -0.15226200 & -1.15009000 \\ \mathrm{H} & -6.28364400 & -0.64086600 & -1.27631200 \\ \mathrm{O} & -3.51648900 & -1.84787800 & -1.40761400 \\ \mathrm{H} & -3.34218500 & -1.93687500 & -2.36000300 \\ \mathrm{H} & -4.69675200 & -0.84854900 & -1.27338900 \\ \mathrm{~N} & 0.63838300 & -0.84964500 & -0.00490300 \\ \mathrm{C} & 1.39384000 & 0.20958200 & -0.21183500 \\ \mathrm{H} & 0.87434900 & 1.14398000 & -0.41724300 \\ \mathrm{C} & 2.79937400 & 0.20134400 & -0.16549400 \\ \mathrm{C} & 3.38306600 & -1.08070700 & 0.14180800 \\ \mathrm{~N} & 2.62612000 & -2.16502500 & 0.32964400 \\ \mathrm{C} & 1.26114400 & -2.08070400 & 0.24170900 \\ \mathrm{O} & 0.54245200 & -3.09707200 & 0.37899700 \\ \mathrm{C} & 3.49201500 & 1.45109100 & -0.43189100 \\ \mathrm{O} & 2.95686400 & 2.52607100 & -0.69917800 \\ \mathrm{O} & 4.85728400 & 1.37019400 & -0.37183500 \\ \mathrm{H} & 5.21086000 & 2.25716300 & -0.57361900 \\ \mathrm{~N} & 4.72047800 & -1.26056700 & 0.27810700 \\ \mathrm{H} & 5.05975900 & -2.20650800 & 0.38835800 \\ \mathrm{H} & 5.37074800 & -0.52562200 & 0.04064800 \\ & & & \\ & & & \end{array}$

Coordinates for the TS along the deglycosylation of dU facilitated by the $\mathrm{HO}^{-} \ldots \mathrm{H}_{2} \mathrm{O}$ nucleophile.

$\begin{array}{llll}\mathrm{C} & -1.91334700 & 2.53742200 & -0.56968800 \\ \mathrm{H} & -1.91731900 & 2.75198600 & -1.64631200 \\ \mathrm{H} & -2.79089800 & 3.02167200 & -0.11961000 \\ \mathrm{C} & -2.06549300 & 1.04691200 & -0.36053300 \\ \mathrm{O} & -1.05459600 & 0.31655900 & -1.13241200 \\ \mathrm{C} & -0.70353000 & -0.78179200 & -0.50382700 \\ \mathrm{H} & -0.22867100 & -1.56194700 & -1.06542600 \\ \mathrm{~N} & 1.38698000 & -0.27452800 & -0.02881100 \\ \mathrm{C} & 1.92053000 & 0.94404000 & -0.26534400 \\ \mathrm{H} & 1.19093400 & 1.72720600 & -0.46454000\end{array}$




$\begin{array}{lrrr}\mathrm{C} & 3.26623900 & 1.23538600 & -0.25136400 \\ \mathrm{C} & 4.20006900 & 0.18148500 & 0.02061100 \\ \mathrm{O} & 5.44457900 & 0.27232600 & 0.06975400 \\ \mathrm{~N} & 3.58797600 & -1.05562100 & 0.24311000 \\ \mathrm{H} & 4.20022700 & -1.84459100 & 0.42808100 \\ \mathrm{C} & 2.21644500 & -1.32634800 & 0.21598600 \\ \mathrm{O} & 1.81616600 & -2.49201200 & 0.41119500 \\ \mathrm{C} & -1.93592900 & 0.50682100 & 1.07507100 \\ \mathrm{C} & -1.24816500 & -0.86466400 & 0.89256600 \\ \mathrm{H} & -0.45757900 & -1.05498700 & 1.61805200 \\ \mathrm{H} & -1.98084900 & -1.67189500 & 0.93865900 \\ \mathrm{H} & -1.32464200 & 1.17954500 & 1.68278700 \\ \mathrm{H} & -3.03170500 & 0.73601600 & -0.77068900 \\ \mathrm{O} & -3.25754100 & 0.43563600 & 1.62111900 \\ \mathrm{O} & -0.70753200 & 2.99998600 & 0.03760300 \\ \mathrm{H} & -3.19516200 & 0.29254100 & 2.57959700 \\ \mathrm{H} & -0.64234700 & 3.95886100 & -0.09433000 \\ \mathrm{O} & -4.75025300 & -1.52250200 & -0.12038300 \\ \mathrm{H} & -4.45504000 & -0.88205100 & 0.55217400 \\ \mathrm{O} & -2.47257000 & -2.10837800 & -1.30432900 \\ \mathrm{H} & -2.50348600 & -1.90920400 & -2.25523200 \\ \mathrm{H} & -3.89123700 & -1.75688400 & -0.62451500 \\ \mathrm{H} & 3.63614300 & 2.23629400 & -0.44002300\end{array}$

Coordinates for the TS along the deglycosylation of $\mathrm{dT}$ facilitated by the $\mathrm{HO}^{-} \cdots \mathrm{H}_{2} \mathrm{O}$ nucleophile.

$\begin{array}{lrrr}\mathrm{C} & -2.01061400 & 2.51173000 & -0.27003500 \\ \mathrm{H} & -2.18184600 & 2.73963000 & -1.33036100 \\ \mathrm{H} & -2.77327500 & 3.03790800 & 0.32057700 \\ \mathrm{C} & -2.21444900 & 1.03158800 & -0.04344100 \\ \mathrm{O} & -1.37443800 & 0.24984200 & -0.96926800 \\ \mathrm{C} & -1.05684500 & -0.89771800 & -0.42244700 \\ \mathrm{H} & -0.63534200 & -1.65898000 & -1.04841700 \\ \mathrm{~N} & 1.10591600 & -0.53859900 & 0.02389300 \\ \mathrm{C} & 1.72938300 & 0.61344200 & -0.32208800 \\ \mathrm{H} & 1.05380700 & 1.43351100 & -0.56257400 \\ \mathrm{C} & 3.09136700 & 0.81880700 & -0.37075600 \\ \mathrm{C} & 3.93690400 & -0.29736900 & -0.03158600 \\ \mathrm{O} & 5.18681900 & -0.30082400 & -0.01896900 \\ \mathrm{~N} & 3.24235100 & -1.46017400 & 0.30394500 \\ \mathrm{H} & 3.80146800 & -2.27579800 & 0.53544800 \\ \mathrm{C} & 1.85484800 & -1.62907500 & 0.32800800 \\ \mathrm{O} & 1.37826900 & -2.74747000 & 0.62043600 \\ \mathrm{C} & -1.87005200 & 0.44883000 & 1.33513200 \\ \mathrm{C} & -1.53330400 & -1.01649900 & 0.99241300 \\ \mathrm{H} & -0.78846200 & -1.48085800 & 1.63766000 \\ \mathrm{H} & -2.44695400 & -1.61740100 & 0.99284600 \\ \mathrm{H} & -0.99906100 & 0.96091000 & 1.75526200 \\ \mathrm{H} & -3.25120600 & 0.77876500 & -0.29278900\end{array}$




$\begin{array}{lrrr}\mathrm{O} & -3.00061400 & 0.62067000 & 2.18444900 \\ \mathrm{O} & -0.69722600 & 2.90208300 & 0.12942200 \\ \mathrm{C} & 3.71256700 & 2.13498000 & -0.75487000 \\ \mathrm{H} & 4.33132700 & 2.54575300 & 0.05414200 \\ \mathrm{H} & 4.36584100 & 2.03557600 & -1.63180200 \\ \mathrm{H} & 2.93797900 & 2.87180600 & -0.99349500 \\ \mathrm{H} & -2.72100000 & 0.49351600 & 3.10493700 \\ \mathrm{H} & -0.59548100 & 3.85281600 & -0.03450100 \\ \mathrm{O} & -5.05148600 & -0.71441600 & -1.08983000 \\ \mathrm{H} & -5.80359000 & -1.32605100 & -1.08073600 \\ \mathrm{O} & -2.89692500 & -2.14887200 & -1.23382500 \\ \mathrm{H} & -2.71948500 & -2.29044900 & -2.17930400 \\ \mathrm{H} & -4.20662700 & -1.30963600 & -1.16306500\end{array}$

Coordinates for the TS along the deglycosylation of $5 \mathrm{hm}$-dU facilitated by the $\mathrm{HO}^{-} \cdots \mathrm{H}_{2} \mathrm{O}$ nucleophile.

$\begin{array}{llrr}\mathrm{C} & -2.03036400 & 2.57349200 & -0.33563800 \\ \mathrm{H} & -2.21384600 & 2.78503200 & -1.39722800 \\ \mathrm{H} & -2.71742500 & 3.18987700 & 0.26037500 \\ \mathrm{C} & -2.37009100 & 1.12857500 & -0.05336500 \\ \mathrm{O} & -1.63900200 & 0.23991100 & -0.97812600 \\ \mathrm{C} & -1.40913800 & -0.91309100 & -0.40256500 \\ \mathrm{H} & -1.08957900 & -1.73130800 & -1.01713300 \\ \mathrm{C} & -2.03897400 & 0.55549600 & 1.33246000 \\ \mathrm{C} & -1.85533900 & -0.94451100 & 1.02620300 \\ \mathrm{H} & -1.14410600 & -1.46265700 & 1.66856300 \\ \mathrm{H} & -2.82280100 & -1.45341800 & 1.06425700 \\ \mathrm{H} & -1.11025500 & 0.99291600 & 1.71160800 \\ \mathrm{H} & -3.43331900 & 0.96998600 & -0.26508200 \\ \mathrm{O} & -3.12015000 & 0.85880600 & 2.20809500 \\ \mathrm{O} & -0.67212400 & 2.84321600 & 0.01028100 \\ \mathrm{H} & -2.82410000 & 0.73306200 & 3.12362700 \\ \mathrm{H} & -0.48348300 & 3.77491500 & -0.18305900 \\ \mathrm{O} & -5.33052800 & -0.31909300 & -1.01796600 \\ \mathrm{H} & -6.15985200 & -0.82063200 & -0.99417900 \\ \mathrm{O} & -3.39593500 & -2.03679800 & -1.12580600 \\ \mathrm{H} & -3.25989700 & -2.25595300 & -2.06322200 \\ \mathrm{H} & -4.57502300 & -1.02725200 & -1.08167300 \\ \mathrm{~N} & 0.78584700 & -0.75532400 & -0.02793100 \\ \mathrm{C} & 1.50329200 & 0.33846700 & -0.36508300 \\ \mathrm{H} & 0.90516700 & 1.21679100 & -0.60592800 \\ \mathrm{C} & 2.87922200 & 0.41028000 & -0.41028300 \\ \mathrm{C} & 3.62041900 & -0.76557900 & -0.04637900 \\ \mathrm{O} & 4.87134300 & -0.84339500 & 0.00590700 \\ \mathrm{~N} & 2.83576500 & -1.86430500 & 0.27862900 \\ \mathrm{H} & 3.31856300 & -2.72048200 & 0.53515600 \\ \mathrm{C} & 1.43592800 & -1.90988400 & 0.28288700 \\ \mathrm{O} & 0.86034100 & -2.97837500 & 0.56896000 \\ \mathrm{C} & 3.64925300 & 1.63593900 & -0.81935600\end{array}$




$\begin{array}{llll}H & 4.15810700 & 1.47068400 & -1.78158600 \\ \mathrm{H} & 2.97554800 & 2.48872200 & -0.94003400 \\ \mathrm{O} & 4.63197800 & 2.02431800 & 0.16022100 \\ \mathrm{H} & 5.17239500 & 1.22328400 & 0.30484300\end{array}$

Coordinates for the TS along the deglycosylation of $\mathrm{dC}$ facilitated by the $\mathrm{HO}^{-} \cdots \mathrm{H}_{2} \mathrm{O}$ nucleophile with a discrete water bound to the 02 (N3) nucleobase site.

$\begin{array}{llll}\mathrm{C} & 2.785563 & -2.272738 & -0.219272 \\ \mathrm{H} & 3.086971 & -2.446784 & -1.260655 \\ \mathrm{H} & 3.620776 & -2.564698 & 0.432130 \\ \mathrm{C} & 2.554829 & -0.793613 & -0.010991 \\ \mathrm{O} & 1.596272 & -0.277880 & -1.003850 \\ \mathrm{C} & 0.941378 & 0.743181 & -0.502792 \\ \mathrm{H} & 0.351109 & 1.345565 & -1.163100 \\ \mathrm{C} & 1.970933 & -0.327914 & 1.331338 \\ \mathrm{C} & 1.262962 & 0.985013 & 0.940383 \\ \mathrm{H} & 0.370813 & 1.217186 & 1.520996 \\ \mathrm{H} & 1.967630 & 1.818939 & 0.999132 \\ \mathrm{H} & 1.251847 & -1.062639 & 1.706124 \\ \mathrm{H} & 3.496649 & -0.264947 & -0.196287 \\ \mathrm{O} & 3.046813 & -0.176721 & 2.253422 \\ \mathrm{O} & 1.607641 & -3.013158 & 0.100156 \\ \mathrm{H} & 2.683281 & -0.127632 & 3.151745 \\ \mathrm{H} & 1.792678 & -3.956743 & -0.028121 \\ \mathrm{O} & 4.850033 & 1.647518 & -0.926749 \\ \mathrm{H} & 5.404970 & 2.442231 & -0.907701 \\ \mathrm{O} & 2.393417 & 2.438746 & -1.201163 \\ \mathrm{H} & 2.236677 & 2.531100 & -2.156375 \\ \mathrm{H} & 3.880428 & 1.986625 & -1.052223 \\ \mathrm{~N} & -1.078398 & -0.201192 & -0.184870 \\ \mathrm{C} & -1.315391 & -1.490932 & -0.457924 \\ \mathrm{H} & -0.434567 & -2.105787 & -0.635559 \\ \mathrm{C} & -2.586173 & -2.035885 & -0.500871 \\ \mathrm{H} & -2.755071 & -3.085718 & -0.714922 \\ \mathrm{C} & -3.649143 & -1.136205 & -0.241439 \\ \mathrm{~N} & -4.952179 & -1.579361 & -0.195595 \\ \mathrm{~N} & -3.442309 & 0.159184 & 0.013029 \\ \mathrm{C} & -2.154299 & 0.631423 & 0.032382 \\ \mathrm{O} & -1.938317 & 1.865604 & 0.250682 \\ \mathrm{H} & -5.172522 & -2.444680 & -0.672431 \\ \mathrm{H} & -5.665667 & -0.863943 & -0.271345 \\ \mathrm{O} & -4.111993 & 3.444065 & 0.601680 \\ \mathrm{H} & -4.885382 & 2.863138 & 0.533360 \\ \mathrm{H} & -3.335292 & 2.828996 & 0.468967\end{array}$

Coordinates for the TS along the deglycosylation of $\mathrm{dC}$ facilitated by the $\mathrm{HO}^{-} \cdots \mathrm{H}_{2} \mathrm{O}$ nucleophile with a discrete water bound to the N3(N4) nucleobase site.

C $\quad-2.806622 \quad 2.247165 \quad-0.452939$ 


$\begin{array}{llll}\mathrm{H} & -3.082212 & 2.332244 & -1.512393 \\ \mathrm{H} & -3.641509 & 2.630344 & 0.149763 \\ \mathrm{C} & -2.635722 & 0.787059 & -0.101617 \\ \mathrm{O} & -1.684250 & 0.144565 & -1.023342 \\ \mathrm{C} & -1.084490 & -0.855362 & -0.418359 \\ \mathrm{H} & -0.511281 & -1.542426 & -1.006916 \\ \mathrm{C} & -2.087865 & 0.430505 & 1.288189 \\ \mathrm{C} & -1.432575 & -0.942723 & 1.036724 \\ \mathrm{H} & -0.558150 & -1.151743 & 1.652068 \\ \mathrm{H} & -2.172872 & -1.736257 & 1.167745 \\ \mathrm{H} & -1.341894 & 1.168000 & 1.600101 \\ \mathrm{H} & -3.595186 & 0.278975 & -0.251193 \\ \mathrm{O} & -3.178458 & 0.415670 & 2.204939 \\ \mathrm{O} & -1.606881 & 2.970821 & -0.179252 \\ \mathrm{H} & -2.825892 & 0.424916 & 3.108992 \\ \mathrm{H} & -1.751427 & 3.903591 & -0.403056 \\ \mathrm{O} & -5.046236 & -1.676099 & -0.721864 \\ \mathrm{H} & -5.617331 & -2.459118 & -0.696680 \\ \mathrm{O} & -2.604902 & -2.524220 & -0.982631 \\ \mathrm{H} & -2.461284 & -2.672335 & -1.932881 \\ \mathrm{H} & -4.084149 & -2.036241 & -0.840924 \\ \mathrm{~N} & 0.971988 & 0.021646 & -0.152831 \\ \mathrm{C} & 1.273119 & 1.284934 & -0.474461 \\ \mathrm{H} & 0.424742 & 1.930592 & -0.697417 \\ \mathrm{C} & 2.567103 & 1.776578 & -0.513955 \\ \mathrm{H} & 2.784051 & 2.808562 & -0.767427 \\ \mathrm{C} & 3.586513 & 0.849202 & -0.191964 \\ \mathrm{~N} & 3.311907 & -0.425469 & 0.113774 \\ \mathrm{C} & 2.001356 & -0.860367 & 0.124401 \\ \mathrm{O} & 1.738958 & -2.064691 & 0.383641 \\ \mathrm{~N} & 4.905414 & 1.232729 & -0.136134 \\ \mathrm{H} & 5.174194 & 2.075554 & -0.627000 \\ \mathrm{H} & 5.596482 & 0.488875 & -0.140932 \\ \mathrm{H} & 4.767073 & -1.474771 & 0.317893 \\ \mathrm{O} & 5.694212 & -1.840028 & 0.400059 \\ \mathrm{H} & 5.787053 & -2.481189 & -0.321902 \\ & & & \end{array}$

Coordinates for the TS along the deglycosylation of $5 \mathrm{~m}-\mathrm{dC}$ facilitated by the $\mathrm{HO}^{-} \cdots \mathrm{H}_{2} \mathrm{O}$ nucleophile with a discrete water bound to the O2(N3) nucleobase site.

$\begin{array}{llll}\text { C } & 2.723795 & -2.374183 & -0.287685 \\ \text { C } & 2.631325 & -0.885349 & -0.044491 \\ \text { C } & 1.166624 & 0.808936 & -0.475825 \\ \text { C } & 2.115597 & -0.397793 & 1.317659 \\ \text { C } & 1.534187 & 0.986700 & 0.966269 \\ O & 1.706377 & -0.264048 & -1.006683 \\ O & 3.213383 & -0.373189 & 2.225532 \\ O & 1.489995 & -3.012321 & 0.041665 \\ O & 5.176358 & 1.398082 & -0.861881\end{array}$




\begin{tabular}{llll}
$\mathrm{O}$ & 2.778876 & 2.343946 & -1.167769 \\
$\mathrm{H}$ & 2.987398 & -2.551148 & -1.338803 \\
$\mathrm{H}$ & 3.541706 & -2.755202 & 0.339191 \\
$\mathrm{H}$ & 0.626196 & 1.481781 & -1.110411 \\
$\mathrm{H}$ & 0.680689 & 1.291519 & 1.570813 \\
$\mathrm{H}$ & 2.319431 & 1.744665 & 1.029916 \\
$\mathrm{H}$ & 1.334114 & -1.068698 & 1.687803 \\
$\mathrm{H}$ & 3.613819 & -0.439320 & -0.235159 \\
$\mathrm{H}$ & 2.868660 & -0.300484 & 3.129702 \\
$\mathrm{H}$ & 1.586107 & -3.965583 & -0.110317 \\
$\mathrm{H}$ & 5.778173 & 2.157537 & -0.831963 \\
$\mathrm{H}$ & 2.634703 & 2.442836 & -2.124286 \\
$\mathrm{H}$ & 4.231247 & 1.795916 & -1.000289 \\
$\mathrm{~N}$ & -0.937159 & 0.082640 & -0.133272 \\
$\mathrm{~N}$ & -4.936641 & -0.829506 & 0.009256 \\
$\mathrm{~N}$ & -3.231315 & 0.726569 & 0.049807 \\
$\mathrm{C}$ & -1.328583 & -1.190785 & -0.286445 \\
$\mathrm{C}$ & -2.650330 & -1.606471 & -0.271773 \\
$\mathrm{C}$ & -3.589249 & -0.553675 & -0.086536 \\
$\mathrm{C}$ & -1.900203 & 1.049640 & 0.020658 \\
$\mathrm{C}$ & -3.056455 & -3.049495 & -0.428514 \\
$\mathrm{O}$ & -1.540855 & 2.267759 & 0.132518 \\
$\mathrm{O}$ & -3.606696 & 3.987035 & 0.455536 \\
$\mathrm{H}$ & -0.526415 & -1.916820 & -0.418101 \\
$\mathrm{H}$ & -5.279151 & -1.685349 & -0.407851 \\
$\mathrm{H}$ & -5.554187 & -0.034349 & -0.105360 \\
$\mathrm{H}$ & -4.394025 & 3.420846 & 0.433727 \\
$\mathrm{H}$ & -2.854704 & 3.338411 & 0.333847 \\
$\mathrm{H}$ & -3.622224 & -3.417387 & 0.438921 \\
$\mathrm{H}$ & -3.684110 & -3.214785 & -1.316358 \\
$\mathrm{H}$ & -2.171360 & -3.685563 & -0.536804 \\
& & & \\
\hline
\end{tabular}

Coordinates for the TS along the deglycosylation of $5 \mathrm{~m}-\mathrm{dC}$ facilitated by the $\mathrm{HO}^{-} \cdots \mathrm{H}_{2} \mathrm{O}$ nucleophile with a discrete water bound to the N3(N4) nucleobase site.

$\begin{array}{llll}\mathrm{N} & 0.823557 & -0.180281 & -0.073133 \\ \mathrm{~N} & 3.108977 & -0.840934 & 0.128295 \\ \mathrm{~N} & 4.837399 & 0.689720 & 0.041872 \\ \mathrm{C} & 1.232523 & 1.081240 & -0.262127 \\ \mathrm{C} & 2.559162 & 1.485895 & -0.258950 \\ \mathrm{C} & 3.488197 & 0.431969 & -0.044244 \\ \mathrm{C} & 1.769852 & -1.167396 & 0.109185 \\ \mathrm{C} & 2.979203 & 2.920177 & -0.454605 \\ \mathrm{O} & 1.407480 & -2.368107 & 0.253263 \\ \mathrm{O} & 5.339777 & -2.503335 & 0.289023 \\ \mathrm{H} & 0.439804 & 1.813643 & -0.416924 \\ \mathrm{H} & 5.190662 & 1.538596 & -0.379220 \\ \mathrm{H} & 5.454416 & -0.113208 & -0.030797 \\ \mathrm{H} & 4.456277 & -2.037481 & 0.236939\end{array}$




$\begin{array}{llll}\text { H } & 5.377826 & -3.077825 & -0.491664 \\ H & 3.548693 & 3.306792 & 0.402336 \\ H & 3.607322 & 3.057072 & -1.347017 \\ H & 2.099718 & 3.561088 & -0.579021 \\ \mathrm{C} & -2.835259 & 2.268879 & -0.470284 \\ \mathrm{C} & -2.749344 & 0.803924 & -0.108102 \\ \mathrm{C} & -1.282634 & -0.920474 & -0.388761 \\ \mathrm{C} & -2.248710 & 0.425284 & 1.293550 \\ \mathrm{C} & -1.665818 & -0.983149 & 1.059179 \\ \mathrm{O} & -1.816115 & 0.107819 & -1.008086 \\ \mathrm{O} & -3.355422 & 0.474714 & 2.189449 \\ \mathrm{O} & -1.602160 & 2.928740 & -0.183573 \\ \mathrm{O} & -5.275861 & -1.526975 & -0.802461 \\ \mathrm{O} & -2.879069 & -2.508462 & -0.977540 \\ \mathrm{H} & -3.089867 & 2.361288 & -1.534354 \\ \mathrm{H} & -3.656921 & 2.701064 & 0.117208 \\ \mathrm{H} & -0.730446 & -1.638829 & -0.959918 \\ \mathrm{H} & -0.818690 & -1.237561 & 1.695130 \\ \mathrm{H} & -2.452339 & -1.733237 & 1.176091 \\ \mathrm{H} & -1.469679 & 1.122234 & 1.617827 \\ \mathrm{H} & -3.731426 & 0.346300 & -0.273257 \\ \mathrm{H} & -3.019457 & 0.468621 & 3.099823 \\ \mathrm{H} & -1.694418 & 3.866605 & -0.413585 \\ \mathrm{H} & -5.884495 & -2.277237 & -0.721776 \\ \mathrm{H} & -2.717280 & -2.679924 & -1.920904 \\ \mathrm{H} & -4.331304 & -1.940786 & -0.885808\end{array}$

Coordinates for the TS along the deglycosylation of $5 \mathrm{ca}-\mathrm{dC}$ facilitated by the $\mathrm{HO}^{-} \cdots \mathrm{H}_{2} \mathrm{O}$ nucleophile with a discrete water bound to the $\mathrm{O} 2$ (N3) nucleobase site.

$\begin{array}{llll}\mathrm{C} & 2.493574 & -2.589132 & 0.201327 \\ \mathrm{H} & 2.646485 & -3.013805 & -0.799408 \\ \mathrm{H} & 3.250038 & -3.016262 & 0.874294 \\ \mathrm{C} & 2.731178 & -1.097970 & 0.150775 \\ \mathrm{O} & 1.899189 & -0.483982 & -0.903182 \\ \mathrm{C} & 1.579416 & 0.739740 & -0.566588 \\ \mathrm{H} & 1.174431 & 1.384117 & -1.321925 \\ \mathrm{C} & 2.401062 & -0.270153 & 1.402181 \\ \mathrm{C} & 2.056349 & 1.107606 & 0.803218 \\ \mathrm{H} & 1.310814 & 1.678816 & 1.355405 \\ \mathrm{H} & 2.965976 & 1.704719 & 0.690462 \\ \mathrm{H} & 1.537329 & -0.697592 & 1.920537 \\ \mathrm{H} & 3.769893 & -0.912374 & -0.143899 \\ \mathrm{O} & 3.543180 & -0.283629 & 2.253488 \\ \mathrm{O} & 1.175519 & -2.861232 & 0.675453 \\ \mathrm{H} & 3.277966 & 0.021928 & 3.135539 \\ \mathrm{H} & 1.034500 & -3.820807 & 0.666154 \\ \mathrm{O} & 5.514484 & 0.332725 & -1.278048 \\ \mathrm{H} & 6.296308 & 0.905547 & -1.299282\end{array}$




$\begin{array}{llll}\mathrm{O} & 3.442452 & 1.850413 & -1.589941 \\ \mathrm{H} & 3.258240 & 1.885191 & -2.543940 \\ \mathrm{H} & 4.701078 & 0.961070 & -1.423226 \\ \mathrm{O} & -2.435073 & 4.906552 & 0.434153 \\ \mathrm{H} & -3.341674 & 4.562136 & 0.432125 \\ \mathrm{H} & -1.869161 & 4.094156 & 0.336560 \\ \mathrm{~N} & -0.586718 & 0.475226 & -0.093545 \\ \mathrm{C} & -1.191320 & -0.688305 & -0.240910 \\ \mathrm{H} & -0.547790 & -1.554825 & -0.379893 \\ \mathrm{C} & -2.584049 & -0.864282 & -0.209608 \\ \mathrm{C} & -3.340786 & 0.343337 & 0.004491 \\ \mathrm{~N} & -2.734123 & 1.529288 & 0.134677 \\ \mathrm{C} & -1.374705 & 1.612385 & 0.071289 \\ \mathrm{O} & -0.796134 & 2.729782 & 0.160537 \\ \mathrm{C} & -3.100211 & -2.213041 & -0.395296 \\ \mathrm{O} & -2.420938 & -3.219162 & -0.586414 \\ \mathrm{O} & -4.463271 & -2.309964 & -0.345705 \\ \mathrm{H} & -4.696145 & -3.247500 & -0.485173 \\ \mathrm{~N} & -4.690031 & 0.356395 & 0.094101 \\ \mathrm{H} & -5.155449 & 1.247712 & 0.196877 \\ \mathrm{H} & -5.235046 & -0.481728 & -0.045836\end{array}$

Coordinates for the TS along the deglycosylation of $5 \mathrm{ca}-\mathrm{dC}$ facilitated by the $\mathrm{HO}^{-} \cdots \mathrm{H}_{2} \mathrm{O}$ nucleophile with a discrete water bound to the N3(N4) nucleobase site.

$\begin{array}{llll}\mathrm{O} & 4.748158 & -3.319789 & 0.527664 \\ \mathrm{H} & 4.848625 & -3.901020 & -0.242577 \\ \mathrm{H} & 3.891668 & -2.829900 & 0.385906 \\ \mathrm{~N} & 0.479570 & -0.536802 & -0.083563 \\ \mathrm{~N} & 2.671282 & -1.488964 & 0.150493 \\ \mathrm{~N} & 4.581237 & -0.237355 & 0.073949 \\ \mathrm{C} & -2.722663 & 2.400459 & 0.202669 \\ \mathrm{C} & -2.898486 & 0.900293 & 0.164586 \\ \mathrm{C} & -1.678703 & -0.894466 & -0.548506 \\ \mathrm{C} & -2.526636 & 0.095500 & 1.419213 \\ \mathrm{C} & -2.131163 & -1.271701 & 0.827096 \\ \mathrm{C} & 1.035051 & 0.645815 & -0.255358 \\ \mathrm{C} & 2.420121 & 0.885303 & -0.238030 \\ \mathrm{C} & 3.232690 & -0.280973 & -0.005825 \\ \mathrm{C} & 1.309398 & -1.648683 & 0.093725 \\ \mathrm{C} & 2.873310 & 2.250496 & -0.453051 \\ \mathrm{O} & -2.049142 & 0.313597 & -0.890760 \\ \mathrm{O} & -3.662804 & 0.069081 & 2.278324 \\ \mathrm{O} & -1.414024 & 2.731369 & 0.665361 \\ \mathrm{O} & -5.618865 & -0.648219 & -1.256559 \\ \mathrm{O} & -3.488634 & -2.089519 & -1.554406 \\ \mathrm{O} & 0.792101 & -2.781954 & 0.199367 \\ \mathrm{O} & 2.148424 & 3.221373 & -0.661537 \\ \mathrm{O} & 4.231255 & 2.411436 & -0.411917 \\ & & & \end{array}$




$\begin{array}{llll}H & -2.899919 & 2.810544 & -0.800144 \\ H & -3.491812 & 2.800688 & 0.877751 \\ H & -1.245833 & -1.524480 & -1.300131 \\ H & -1.358444 & -1.808021 & 1.376886 \\ H & -3.016988 & -1.905473 & 0.726321 \\ H & -1.677219 & 0.560565 & 1.928821 \\ H & -3.930731 & 0.670013 & -0.121418 \\ H & -3.379553 & -0.217746 & 3.161030 \\ H & -1.316978 & 3.696358 & 0.656789 \\ H & -6.378470 & -1.250166 & -1.278066 \\ H & -3.301697 & -2.123412 & -2.507975 \\ H & -4.782605 & -1.245532 & -1.396976 \\ H & 0.355618 & 1.482950 & -0.404384 \\ H & 4.418893 & 3.355910 & -0.571196 \\ H & 5.089693 & -1.107084 & 0.195241 \\ H & 5.081865 & 0.626402 & -0.075434\end{array}$

Coordinates for the TS along the deglycosylation of $\mathrm{dT}$ facilitated by the $\mathrm{HO}^{-} \cdots \mathrm{H}_{2} \mathrm{O}$ nucleophile with a discrete water bound to the 02 (N3) nucleobase site.

$\begin{array}{llll}\mathrm{C} & 2.704813 & -2.360659 & -0.049765 \\ \mathrm{H} & 2.969771 & -2.634565 & -1.079481 \\ \mathrm{H} & 3.516028 & -2.693378 & 0.612453 \\ \mathrm{C} & 2.630444 & -0.855502 & 0.059343 \\ \mathrm{O} & 1.713996 & -0.311148 & -0.962031 \\ \mathrm{C} & 1.162768 & 0.792689 & -0.525040 \\ \mathrm{H} & 0.651388 & 1.417876 & -1.230036 \\ \mathrm{C} & 2.112303 & -0.242622 & 1.368934 \\ \mathrm{C} & 1.540072 & 1.106237 & 0.889485 \\ \mathrm{H} & 0.696244 & 1.481566 & 1.467205 \\ \mathrm{H} & 2.332926 & 1.859489 & 0.865789 \\ \mathrm{H} & 1.324665 & -0.871647 & 1.794777 \\ \mathrm{H} & 3.616752 & -0.436579 & -0.169686 \\ \mathrm{O} & 3.206670 & -0.139544 & 2.274379 \\ \mathrm{O} & 1.461398 & -2.949306 & 0.330512 \\ \mathrm{H} & 2.859409 & -0.003742 & 3.170240 \\ \mathrm{H} & 1.539751 & -3.913266 & 0.254763 \\ \mathrm{O} & 5.127303 & 1.255059 & -1.071536 \\ \mathrm{H} & 5.767282 & 1.980450 & -1.007985 \\ \mathrm{O} & 2.790502 & 2.322193 & -1.379267 \\ \mathrm{H} & 2.632303 & 2.392034 & -2.336057 \\ \mathrm{H} & 4.203605 & 1.706207 & -1.213023 \\ \mathrm{~N} & -0.919084 & 0.084780 & -0.127036 \\ \mathrm{C} & -1.314887 & -1.196092 & -0.338885 \\ \mathrm{H} & -0.496577 & -1.900698 & -0.480260 \\ \mathrm{C} & -2.615864 & -1.645528 & -0.366462 \\ \mathrm{C} & -3.655783 & -0.668571 & -0.157169 \\ \mathrm{~N} & -3.190751 & 0.632268 & 0.042208 \\ \mathrm{C} & -1.859271 & 1.037487 & 0.049028\end{array}$




$\begin{array}{llll}\mathrm{O} & -1.585182 & 2.257391 & 0.215881 \\ \mathrm{O} & -3.945750 & 3.604434 & 0.475956 \\ \mathrm{H} & -4.075414 & 3.905371 & 1.388887 \\ \mathrm{H} & -3.015250 & 3.262849 & 0.433434 \\ \mathrm{O} & -4.884350 & -0.891233 & -0.143575 \\ \mathrm{C} & -2.985428 & -3.085972 & -0.597443 \\ \mathrm{H} & -3.621022 & -3.204320 & -1.484920 \\ \mathrm{H} & -2.087680 & -3.696681 & -0.740989 \\ \mathrm{H} & -3.546167 & -3.501961 & 0.249984 \\ \mathrm{H} & -3.890859 & 1.360086 & 0.183994\end{array}$

Coordinates for the TS along the deglycosylation of $\mathrm{dT}$ facilitated by the $\mathrm{HO}^{-} \cdots \mathrm{H}_{2} \mathrm{O}$ nucleophile with a discrete water bound to the 04 (N3) nucleobase site.

$\begin{array}{llll}\mathrm{C} & -2.299502 & 2.596780 & -0.286617 \\ \mathrm{H} & -2.465048 & 2.833651 & -1.345879 \\ \mathrm{H} & -3.014866 & 3.179111 & 0.310333 \\ \mathrm{C} & -2.609347 & 1.136595 & -0.051821 \\ \mathrm{O} & -1.835696 & 0.291580 & -0.982387 \\ \mathrm{C} & -1.592544 & -0.871650 & -0.433148 \\ \mathrm{H} & -1.231443 & -1.662253 & -1.060478 \\ \mathrm{C} & -2.295648 & 0.533341 & 1.325052 \\ \mathrm{C} & -2.073523 & -0.953517 & 0.982274 \\ \mathrm{H} & -1.367726 & -1.475819 & 1.627220 \\ \mathrm{H} & -3.031287 & -1.481763 & 0.982148 \\ \mathrm{H} & -1.384258 & 0.979205 & 1.735045 \\ \mathrm{H} & -3.663496 & 0.958605 & -0.292006 \\ \mathrm{O} & -3.401331 & 0.790458 & 2.184682 \\ \mathrm{O} & -0.956505 & 2.889986 & 0.097209 \\ \mathrm{H} & -3.122516 & 0.645706 & 3.102792 \\ \mathrm{H} & -0.790903 & 3.833547 & -0.055492 \\ \mathrm{O} & -5.518878 & -0.394179 & -1.106575 \\ \mathrm{H} & -6.328374 & -0.927625 & -1.111445 \\ \mathrm{O} & -3.519110 & -2.031346 & -1.240058 \\ \mathrm{H} & -3.355418 & -2.203545 & -2.182876 \\ \mathrm{H} & -4.735347 & -1.070686 & -1.179737 \\ \mathrm{~N} & 0.591033 & -0.672531 & 0.011704 \\ \mathrm{C} & 1.298689 & 0.440143 & -0.286092 \\ \mathrm{H} & 0.688583 & 1.309479 & -0.528331 \\ \mathrm{C} & 2.674483 & 0.555281 & -0.286882 \\ \mathrm{C} & 3.423755 & -0.620548 & 0.054438 \\ \mathrm{~N} & 2.650841 & -1.739704 & 0.339357 \\ \mathrm{C} & 1.254730 & -1.817922 & 0.316476 \\ \mathrm{O} & 0.697186 & -2.905987 & 0.569942 \\ \mathrm{O} & 7.013686 & 0.717297 & -0.202625 \\ \mathrm{H} & 6.846940 & 1.618475 & -0.518751 \\ \mathrm{H} & 6.124309 & 0.298010 & -0.119445 \\ \mathrm{O} & 4.674789 & -0.720770 & 0.113604 \\ \mathrm{C} & 3.389427 & 1.837074 & -0.621749\end{array}$




$\begin{array}{llll}\mathrm{H} & 4.009331 & 2.189353 & 0.213282 \\ \mathrm{H} & 4.053914 & 1.719279 & -1.487911 \\ \mathrm{H} & 2.668938 & 2.626847 & -0.859252 \\ \mathrm{H} & 3.149304 & -2.593683 & 0.571412\end{array}$

Coordinates for the TS along the deglycosylation of $5 \mathrm{hm}$-dU facilitated by the $\mathrm{HO}^{-} \cdot \ldots \mathrm{H}_{2} \mathrm{O}$ nucleophile with a discrete water bound to the $\mathrm{O} 2$ (N3) nucleobase site.

$\begin{array}{llll}\mathrm{O} & -3.317375 & 4.135004 & 0.538822 \\ \mathrm{H} & -3.437232 & 4.438355 & 1.452357 \\ \mathrm{H} & -2.411647 & 3.735453 & 0.502534 \\ \mathrm{~N} & -0.675799 & 0.350027 & -0.138062 \\ \mathrm{~N} & -2.874359 & 1.166219 & 0.021677 \\ \mathrm{C} & 2.586321 & -2.526310 & 0.040370 \\ \mathrm{C} & 2.721554 & -1.023180 & 0.108162 \\ \mathrm{C} & 1.476935 & 0.785162 & -0.525916 \\ \mathrm{C} & 2.292084 & -0.309346 & 1.398479 \\ \mathrm{C} & 1.889759 & 1.085756 & 0.880174 \\ \mathrm{C} & -1.212213 & -0.865296 & -0.395936 \\ \mathrm{C} & -2.558990 & -1.141576 & -0.466628 \\ \mathrm{C} & -3.476466 & -0.060848 & -0.224797 \\ \mathrm{C} & -1.500978 & 1.406429 & 0.053297 \\ \mathrm{C} & -3.128542 & -2.496975 & -0.786170 \\ \mathrm{O} & 1.890243 & -0.386241 & -0.934390 \\ \mathrm{O} & 3.397923 & -0.316753 & 2.295947 \\ \mathrm{O} & 1.272139 & -2.925754 & 0.428381 \\ \mathrm{O} & 5.401762 & 0.626561 & -1.188447 \\ \mathrm{O} & 3.293947 & 2.108701 & -1.413640 \\ \mathrm{O} & -4.725324 & -0.166210 & -0.210607 \\ \mathrm{O} & -1.085504 & 2.575454 & 0.259411 \\ \mathrm{O} & -4.080360 & -2.942208 & 0.198548 \\ \mathrm{H} & 2.813243 & -2.862638 & -0.979774 \\ \mathrm{H} & 3.341608 & -2.949407 & 0.716927 \\ \mathrm{H} & 1.061268 & 1.454910 & -1.253028 \\ \mathrm{H} & 1.099918 & 1.579260 & 1.445313 \\ \mathrm{H} & 2.769341 & 1.734761 & 0.835345 \\ \mathrm{H} & 1.435577 & -0.820579 & 1.848532 \\ \mathrm{H} & 3.755176 & -0.748983 & -0.131000 \\ \mathrm{H} & 3.077449 & -0.108936 & 3.188030 \\ \mathrm{H} & 1.216508 & -3.893014 & 0.379860 \\ \mathrm{H} & 6.166233 & 1.219097 & -1.123084 \\ \mathrm{H} & 3.130800 & 2.222445 & -2.365369 \\ \mathrm{H} & 4.574426 & 1.244568 & -1.298307 \\ \mathrm{H} & -0.482719 & -1.660443 & -0.543271 \\ \mathrm{H} & -3.482626 & 1.968528 & 0.192194 \\ \mathrm{H} & -3.618325 & -2.486664 & -1.771972 \\ \mathrm{H} & -2.335193 & -3.248835 & -0.815262 \\ \mathrm{H} & -4.739091 & -2.222934 & 0.256254\end{array}$


Coordinates for the TS along the deglycosylation of $5 \mathrm{hm}$-dU facilitated by the $\mathrm{HO}^{-} \cdots \mathrm{H}_{2} \mathrm{O}$ nucleophile with a discrete water bound to the $\mathrm{O} 4$ (N3) nucleobase site.

$\begin{array}{llll}\mathrm{O} & 5.531105 & -2.434511 & 0.518133 \\ \mathrm{H} & 5.390445 & -1.501606 & 0.221066 \\ \mathrm{H} & 5.944472 & -2.892135 & -0.230669 \\ \mathrm{~N} & 0.527069 & -0.467470 & -0.078960 \\ \mathrm{~N} & 2.727429 & -1.268980 & 0.145257 \\ \mathrm{C} & -2.734167 & 2.412960 & -0.091610 \\ \mathrm{C} & -2.869962 & 0.916008 & 0.061146 \\ \mathrm{C} & -1.623029 & -0.926039 & -0.459659 \\ \mathrm{C} & -2.449655 & 0.277456 & 1.393395 \\ \mathrm{C} & -2.045589 & -1.145733 & 0.958672 \\ \mathrm{C} & 1.062542 & 0.724340 & -0.411435 \\ \mathrm{C} & 2.412722 & 1.000023 & -0.493962 \\ \mathrm{C} & 3.319660 & -0.060899 & -0.177722 \\ \mathrm{C} & 1.350483 & -1.520433 & 0.185214 \\ \mathrm{C} & 2.981511 & 2.332752 & -0.897962 \\ \mathrm{O} & -2.030590 & 0.221911 & -0.936551 \\ \mathrm{O} & -3.560548 & 0.338042 & 2.282600 \\ \mathrm{O} & -1.422230 & 2.834321 & 0.280764 \\ \mathrm{O} & -5.554986 & -0.817988 & -1.128513 \\ \mathrm{O} & -3.427272 & -2.281428 & -1.287975 \\ \mathrm{O} & 4.578582 & 0.054077 & -0.165607 \\ \mathrm{O} & 0.946979 & -2.666948 & 0.458732 \\ \mathrm{O} & 3.906488 & 2.859913 & 0.072433 \\ \mathrm{H} & -2.954585 & 2.690289 & -1.130791 \\ \mathrm{H} & -3.493441 & 2.874523 & 0.554680 \\ \mathrm{H} & -1.196649 & -1.634476 & -1.142351 \\ \mathrm{H} & -1.259107 & -1.605724 & 1.555950 \\ \mathrm{H} & -2.925480 & -1.795624 & 0.946936 \\ \mathrm{H} & -1.595161 & 0.813201 & 1.818104 \\ \mathrm{H} & -3.902272 & 0.628557 & -0.168599 \\ \mathrm{H} & -3.245394 & 0.179305 & 3.186586 \\ \mathrm{H} & -1.365310 & 3.796797 & 0.173541 \\ \mathrm{H} & -6.309363 & -1.417637 & -1.023055 \\ \mathrm{H} & -3.258288 & -2.425012 & -2.234632 \\ \mathrm{H} & -4.719214 & -1.428400 & -1.209152 \\ \mathrm{H} & 0.334401 & 1.508723 & -0.614794 \\ \mathrm{H} & 3.348740 & -2.048866 & 0.362816 \\ \mathrm{H} & 3.494400 & 2.255642 & -1.868677 \\ \mathrm{H} & 2.183552 & 3.073297 & -0.999447 \\ \mathrm{H} & 4.579444 & 2.162588 & 0.189824\end{array}$


Full citation for references 17, 27, and 60:

17. He, Y.-F.; Li, B.-Z.; Li, Z.; Liu, P.; Wang, Y.; Tang, Q.; Ding, J.; Jia, Y.; Chen, Z.; Li, L.; Sun, Y.; Li, X.; Dai, Q.; Song, C.-X.; Zhang, K.; He, C.; Xu, G.-L., TET-mediated formation of 5carboxylcytosine and its excision by TDG in mammalian DNA. Science 2011, 333 (6047), 13031307.

27. Pfaffeneder, T.; Spada, F.; Wagner, M.; Brandmayr, C.; Laube, S. K.; Eisen, D.; Truss, M.; Steinbacher, J.; Hackner, B.; Kotljarova, O.; Schuermann, D.; Michalakis, S.; Kosmatchev, O.; Schiesser, S.; Steigenberger, B.; Raddaoui, N.; Kashiwazaki, G.; Müller, U.; Spruijt, C. G.; Vermeulen, M.; Leonhardt, H.; Schär, P.; Müller, M.; Carell, T., TET oxidizes thymine to 5hydroxymethyluracil in mouse embryonic stem cell DNA. Nature Chem. Biol. 2014, 10 (7), 574581.

60. Frisch, M. J.; Trucks, G. W.; Schlegel, H. B.; Scuseria, G. E.; Robb, M. A.; Cheeseman, J. R.; Scalmani, G.; Barone, V.; Mennucci, B.; Petersson, G. A.; Nakatsuji, H.; Caricato, M.; Li, X.; Hratchian, H. P.; Izmaylov, A. F.; Bloino, J.; Zheng, G.; Sonnenberg, J. L.; Hada, M.; Ehara, M.; Toyota, K.; Fukuda, R.; Hasegawa, J.; Ishida, M.; Nakajima, T.; Honda, Y.; Kitao, O.; Nakai, H.; Vreven, T.; Montgomery Jr., J. A.; Peralta, J. E.; Ogliaro, F.; Bearpark, M. J.; Heyd, J.; Brothers, E. N.; Kudin, K. N.; Staroverov, V. N.; Kobayashi, R.; Normand, J.; Raghavachari, K.; Rendell, A. P.; Burant, J. C.; Iyengar, S. S.; Tomasi, J.; Cossi, M.; Rega, N.; Millam, N. J.; Klene, M.; Knox, J. E.; Cross, J. B.; Bakken, V.; Adamo, C.; Jaramillo, J.; Gomperts, R.; Stratmann, R. E.; Yazyev, O.; Austin, A. J.; Cammi, R.; Pomelli, C.; Ochterski, J. W.; Martin, R. L.; Morokuma, K.; Zakrzewski, V. G.; Voth, G. A.; Salvador, P.; Dannenberg, J. J.; Dapprich, S.; Daniels, A. D.; Farkas, Ö.; Foresman, J. B.; Ortiz, J. V.; Cioslowski, J.; Fox, D. J. Gaussian 09, Revisions C.01 and D.01; Gaussian, Inc.: Wallingford, CT, USA, 2009. 